\title{
Vacuum polarization and classical self-action near higher-dimensional defects
}

\author{
Yuri V. Grats ${ }^{\mathrm{a}}$, Pavel Spirin ${ }^{\mathrm{b}}$ \\ Department of Theoretical Physics, Faculty of Physics, Moscow State University, Moscow 119991, Russian Federation
}

Received: 28 November 2016 / Accepted: 25 January 2017 / Published online: 14 February 2017

(C) The Author(s) 2017. This article is published with open access at Springerlink.com

\begin{abstract}
We analyze the gravity-induced effects associated with a massless scalar field in a higher-dimensional spacetime being the tensor product of $(d-n)$-dimensional Minkowski space and $n$-dimensional spherically/cylindrically symmetric space with a solid/planar angle deficit. These spacetimes are considered as simple models for a multidimensional global monopole (if $n \geqslant 3$ ) or cosmic string (if $n=2)$ with $(d-n-1)$ flat extra dimensions. Thus, we refer to them as conical backgrounds. In terms of the angular-deficit value, we derive the perturbative expression for the scalar Green function, valid for any $d \geqslant 3$ and $2 \leqslant n \leqslant d-1$, and compute it to the leading order. With the use of this Green function we compute the renormalized vacuum expectation value of the field square $\left\langle\phi^{2}(x)\right\rangle_{\text {ren }}$ and the renormalized vacuum averaged of the scalar-field energy-momentum tensor $\left\langle T_{M N}(x)\right\rangle_{\text {ren }}$ for arbitrary $d$ and $n$ from the interval mentioned above and arbitrary coupling constant to the curvature $\xi$. In particular, we revisit the computation of the vacuum polarization effects for a non-minimally coupled massless scalar field in the spacetime of a straight cosmic string. The same Green function enables to consider the old purely classical problem of the gravity-induced self-action of a classical point-like scalar or electric charge, placed at rest at some fixed point of the space under consideration. To deal with divergences, which appear in consideration of the two problems, we apply the dimensional-regularization technique, widely used in quantum field theory. The explicit dependence of the results upon the dimensionalities of both the bulk and conical submanifold is discussed.
\end{abstract}

\section{Introduction}

In the last decades the higher-dimensional generalizations of known four-dimensional solutions in general relativity (GR)

\footnotetext{
a e-mail: grats@phys.msu.ru

b e-mail: pspirin@physics.uoc.gr
}

became the object of intense research in the context of widely developing higher-dimensional theories. It is enough to mention the possibility of the mini-black-hole creation in the high energy physics experiments [1,2]. Experimental confirmation of such a creation is considered as one of the tests of the existence of extra dimensions, or it has to set new bounds on the parameters of the multidimensional theories predicting the existence of mini-black holes. Though at present there are no confirmations of the existence of extra dimensions [3], the modern theories stimulated the research of the GR in $d>4$ spacetime dimensions. This implies not only the search of new solutions, but also the research of the higherdimensional generalizations of the known four-dimensional solutions. The partial goal of such research is to clarify which predictions by GR are proper for four dimensions only, and which ones are universal and extended to higher dimensions. At the other hand, it is expected that the research of higher-dimensional generalizations allows one to shed light on some peculiarities of the standard four-dimensional theory and assists in the better understanding of the latter. This research assumes not only the study of geometric features of higher-dimensional solutions, but also the study of particularities of the classical/quantum matter dynamics on their background.

The standard problems of research within the field theory on the curved background, to which the physicists return through decades, are the effects of the induced by gravity vacuum polarization and the problem of self-action of the classical charged particle. These two problems, weakly related at first glance, in fact have a number of common features. The main of those is that the two problems are determined by the appropriate Green function being the solution of a partial differential equation which is sensitive to the global structure of the manifold. Thus, the two effects become essentially nonlocal. Furthermore, for the elimination of divergences arising in the two cases, one uses the same techniques. 
The present work is devoted to the consideration of gravity-induced effects of the vacuum polarization of a massless scalar field and the self-action of a scalar or electric charge on the ultrastatic spacetime being the product of $(d-n)$-dimensional Minkowski spacetime and $n$ dimensional spherically symmetric space with an angular deficit.

For the particular cases such problems were considered in a series of papers: the analysis of the vacuum polarization in four, five, and six dimensions was done in Refs. [4] (nonperturbative), [5], and [5-7], respectively. The self-action for different four-dimensional models was considered in work by Bezerra de Mello et al. [8,9]. For the cosmic strings the average vacuum energy was considered by Dowker [10] in terms of Bernoulli polynomials for even spacetime dimensionality; the energy-momentum tensor in four dimensions was computed in [11] for the conformal coupling and generalized in [12] to the arbitrary coupling $\xi$, in three-dimensional gravity these problems were discussed in $[10,13]$. The electrostatic self-action near the four-dimensional cosmic string is computed in the integral form in [14]. The basic difficulty to generalize these results is that they are based on the coordinate-space Green function which has a drastically different behavior in odd and even dimensions and has no universal form even for the series of the same spacetime parity.

We will concentrate on the computation of the renormalized vacuum expectation values (VEV) for $\left\langle\phi^{2}(x)\right\rangle_{\text {ren }}$ and $\left\langle T_{M N}(x)\right\rangle_{\text {ren }}$, as well as the calculation of the renormalized self-energy $U_{\text {ren }}(x)$ and self-force $F_{\text {ren }}(x)$ of the static scalar or electric charge. The basic goal here is to obtain the universal expressions valid for given arbitrary $d, n$, and $\xi$, as well as to compare the particular examples with known results. For the regularization of formally diverging expressions we will use the dimensional-regularization technique.

The paper is organized as follows: the Introduction is the first section. In the second section, the Setup, we briefly present the background metric with angular deficit in arbitrary spacetime dimension and derive the initial expressions for the subsequent computation of classical self-force and vacuum averages. The perturbation theory we use is described in Sect. 3, where we also construct the approximate Green function. Section 4 is devoted to the computation of the renormalized vacuum averaged expression $\left\langle\phi^{2}(x)\right\rangle_{\text {ren }}$ in the dimensional-regularization scheme. The comparison with the analogous results known in the literature is presented. The renormalized stress-energy tensor is computed in Sect. 5. The classical self-energy and self-force of a pointlike scalar or electric charge in the spacetime at hand are computed in Sect. 6. In Sect. 7 we discuss the special case of an infinitely thin cosmic string. We show that there is a some ambiguity in the previous calculations and propose an alternative approach to the problem. In Sect. 8, the Conclu- sion, we summarize the results and present prospects. Useful integrals are given in the appendix.

We use the units $G=c=\hbar=1$ and metric with the signature $(-,+,+, \ldots,+)$.

\section{Setup}

In the model we consider quantized or classical massless scalar field $\phi$, living in the static $d$-dimensional bulk with $n$ dimensional submanifold with solid or planar angular deficit. This $n$-dimensional subspace may be considered as created by the $n$-dimensional global monopole (for $n \geqslant 3$ ) or as a straight cosmic string (for $n=2$ ).

First we overview the background geometry.

\subsection{Background of the cosmic string and the global} monopole, and their higher-dimensional analogues

The metric of a straight infinitely thin cosmic string with a mass per unit length $\mu$, located along the $z$-axis in four spacetime dimensions, in cylindric coordinates reads

$\mathrm{d} s^{2}=-\mathrm{d} t^{2}+\mathrm{d} z^{2}+\mathrm{d} \rho^{2}+\beta^{2} \rho^{2} \mathrm{~d} \varphi^{2}$

where $\beta=1-4 G \mu$. (For the review of the formation, evolution and geometry of topological defects and some physical effects near them see $[15,16]$ and the references therein.) The corresponding Riemann tensor vanishes everywhere except for the symmetry axis $\rho=0$, where it has a $\delta$-like singularity [17]. A straight string does not affect the local geometry of the spacetime, its effect on matter fields is purely topological, and the dimensionless parameter $G \mu$ is the only parameter which measures the effect of the conical structure on the dynamics of classical and quantized matter.

In some applications it is more appropriate to use coordinates $(t, x, y, z)$, which are conformally Cartesian on the plane transverse to the string. With the radial-coordinate transformation $\rho \rightarrow r$

$\rho=\frac{r_{0}}{\beta}\left(\frac{r}{r_{0}}\right)^{\beta}, \quad x^{1}=r \cos \varphi, \quad x^{2}=r \sin \varphi$,

where $r_{0}$ is an arbitrary scale with the length dimensionality, the line element (2.1) takes the form

$\mathrm{d} s^{2}=-\mathrm{d} t^{2}+\mathrm{d} z^{2}+\mathrm{e}^{-2(1-\beta) \ln \left(r / r_{0}\right)} \delta_{a b} \mathrm{~d} x^{a} \mathrm{~d} x^{b}$,

where $r^{2}=\delta_{a b} x^{a} x^{b}, a, b=1,2$.

The idea to use conformal coordinates was put forward in the framework of low-dimensional gravity [18-21]. In this case it gives the possibility to find a self-consistent solution for the metric of a multi-center space, i.e. a static $(2+1)$ dimensional spacetime of $N$ point masses. Later it was shown 
that the line element of a multi-center spacetime can be generalized for the case of $N$ parallel cosmic strings [22]. The same idea enables one to obtain the explicit solutions of the problem of topological self-action in the multi-center and multi-string spacetimes [23-26], and it provides an appropriate framework for consideration of the vacuum polarization effect in the spacetime of multiple cosmic strings and, in particular, the vacuum Casimir-like interaction of parallel strings [27].

One can consider the generalization of the metric (2.1) and (2.2) for a spherically symmetric case, when any plane containing the center of symmetry and dividing the space into two equal parts is a cone with the angular deficit $\delta \varphi=$ $2 \pi(1-\beta)$,

$\mathrm{d} s^{2}=-\mathrm{d} t^{2}+\mathrm{d} \varrho^{2}+\beta^{2} \varrho^{2}\left(\mathrm{~d} \theta^{2}+\sin ^{2} \theta \mathrm{d} \varphi^{2}\right)$.

This metric describes an ultrastatic spherically symmetric spacetime with the solid angle deficit equal to $4 \pi\left(1-\beta^{2}\right)$.

Equation (2.3) approximates the metric of a global monopole $[28,29]$. Strictly speaking, the metric of a global monopole contains a mass term, but this term is too small to be of importance on astrophysical scales.

As in the string case, there is a possibility to use conformally Cartesian coordinates on the section $t=$ const of the spacetime (2.3). After a redefinition of the radial coordinate $\beta \varrho=r_{0}\left(r / r_{0}\right)^{\beta}$ the metric of the spatial sector of the above line element takes the conformally Euclidean form. Thus, we can introduce a set of Cartesian coordinates $\left\{x^{i}\right\}, i=1,2,3$ with the usual relation with the spherical coordinates $r, \theta, \varphi$. In these coordinates the metric (2.3) reduces to the form

$\mathrm{d} s^{2}=-\mathrm{d} t^{2}+\mathrm{e}^{-2(1-\beta) \ln \left(r / r_{0}\right)} \delta_{i k} \mathrm{~d} x^{i} \mathrm{~d} x^{k}$,

where $r^{2}=\delta_{i k} x^{i} x^{k}, i, k=1,2,3$.

We see that the two conical defects have no Newtonian potential and exert no gravitational force on the surrounding matter. For the two defects their gravitational properties are determined by the deficit angle only. The main difference of a global monopole from the case of a cosmic string is that the monopole spacetime is not locally flat, and its gravitational field provides a tidal acceleration which is proportional to $r^{-2 \beta}$.

Below we will consider multidimensional generalization of the spaces (2.2) and (2.4), with arbitrary number of conical and flat spatial dimensions. The corresponding metric reads

$$
\begin{aligned}
\mathrm{d} s^{2} & \equiv g_{M N} \mathrm{~d} x^{M} \mathrm{~d} x^{N} \\
& =-\mathrm{d} t^{2}+\mathrm{d} x_{d-1}^{2}+\cdots+\mathrm{d} x_{n+1}^{2}+\mathrm{e}^{-2(1-\beta) \ln r} \delta_{i k} \mathrm{~d} x^{i} \mathrm{~d} x^{k},
\end{aligned}
$$

with $r^{2} \equiv \delta_{i k} x^{i} x^{k}$ and $i, k, \ldots=1, \ldots, n$ while $M, N, \ldots=$ $0,1, \ldots, d-1$. Here $d \geqslant 3$ and $2 \leqslant n \leqslant d-1$. Without loss of generality we put $r_{0}$ equal to unity.
The spacetime with metric (2.5) represents the tensor product of the $(d-n)$-dimensional Minkowski space and the $n$-dimensional centro-symmetric conformally flat space with a solid angle deficit equal to $\delta \Omega=2(1-$ $\left.\beta^{n-1}\right) \pi^{n / 2} / \Gamma(n / 2)$, if $n \geqslant 3$, or planar angular deficit equal to $\delta \varphi=2 \pi(1-\beta)$, if $n=2$.

The corresponding Ricci tensor and the scalar curvature are determined by the conical sector only:

$$
\begin{array}{ll}
R_{i k}=2 \pi(1-\beta) \delta^{2}(\mathbf{r}) \delta_{i k}, & n=2 ; \\
R=4 \pi(1-\beta) r^{2(1-\beta)} \delta^{2}(\mathbf{r}), & \\
R_{i k}=\left(1-\beta^{2}\right)(n-2) \frac{r^{2} \delta_{i k}-x_{i} x_{k}}{r^{4}}, & \\
R=\left(1-\beta^{2}\right) \frac{(n-1)(n-2)}{r^{2 \beta}}, & n \geqslant 3 .
\end{array}
$$

For these spaces and corresponding Green functions we will use the notations $(d, n)$ and $G\left(x, x^{\prime} \mid d, n\right)$. Notice, in these notations, the spacetime of a straight infinitely thin cosmic string and notice that one of the point global monopoles in four spacetime dimensions has the type $(4,2)$ and $(4,3)$, respectively.

Therefore, (2.5) represents the multidimensional generalization of the four-dimensional solutions obtained in [28-31], respectively.

For the first time a metric of the form (2.5) with twodimensional conical subspace $(n=2)$ was considered in Ref. [32]. Later a number of solutions for a coupled system of the Einstein equation and the equations of motion for $n$ scalars was found and analyzed in [33]. It was shown that the $n \geqslant 3$ solution with a zero cosmological constant has approximately the form (2.5) (in our coordinates). Thus, the metric (2.5) describes the conical defects which live in a $d$ dimensional bulk, having a flat $(d-n-1)$-brane as a core. Some tiny QFT effects have been found on these backgrounds for some particular dimensionalities of the bulk dimension $d$ and the dimension of the conical subspace $n$. The vacuum polarization effects for a massless scalar and fermionic fields on the higher-dimensional monopole/string spacetime were investigated in [5-7] and [27,32,34-36]. In [37-39] the authors analyze the vacuum fluctuations of a quantum bosonic and fermionic currents induced by a magnetic flux running along the string. In this paper we continue the investigation of quantum and classical field-theoretical processes on the generalized background (2.5).

The geometry of the spacetime under consideration is simple enough and the metric does not contain any dimensional parameters. Nevertheless we cannot compute explicitly the Green function $G\left(x, x^{\prime} \mid d, n\right)$ in a workable closed form. So, we restrict our consideration by the particular case of a small angular deficit; in the following, we put $(1-\beta) \ll 1$. It enables us to obtain perturbatively the universal expression 
for the Green function, which is valid for any $d$ and $n$ and for any value of the coupling constant $\xi$.

2.2 Self-energy of a point-like charge in a static spacetime: formalism

Let us consider a massless scalar field $\phi$ with a source $j$ in a static $d$-dimensional spacetime with the metric

$\mathrm{d} s^{2} \equiv g_{M N} \mathrm{~d} x^{M} \mathrm{~d} x^{N}=g_{00} \mathrm{~d} t^{2}+g_{\mu \nu} \mathrm{d} x^{\mu} \mathrm{d} x^{\nu}, \quad g_{00}<0$.

In this subsection the small Greek indices $\mu, v, \ldots$ run over all spatial coordinates $1,2, \ldots, d-1$.

The interaction of scalar field with the bulk curvature $R$ is introduced via coupling $\xi$, while the interaction with charges is introduced by the charge density $j(x)$ in a standard way:

$S_{\mathrm{tot}}=-\frac{1}{2} \int d^{d} x \sqrt{-g}\left(\phi_{; M} \phi^{; M}+\xi R \phi^{2}-2 \phi j\right)+S_{j}$.

$S_{j}$ is the action for a charged matter.

From (2.8) one derives the equation of motion for the scalar field:

$\partial_{M}\left(\sqrt{-g} g^{M N} \partial_{N} \phi\right)-\xi \sqrt{-g} \phi R=-\sqrt{-g} j$.

In the static case, when $\partial_{0} \phi=0=\partial_{0} g_{M N}$, and point-like charge $q$ placed at a fixed spatial point $x$ it reads

$\partial_{\mu}\left(\sqrt{-g} g^{\mu \nu} \partial_{\nu} \phi\right)-\xi R \phi \sqrt{-g}=-\sqrt{-g} j$,

where

$j\left(x^{\prime}\right)=q \frac{\delta^{d-1}\left(x-x^{\prime}\right)}{\sqrt{-g}}$.

The field energy in a static spacetime reads

$U=-\int T_{0}^{0} \sqrt{-g} d^{d-1} x$,

where $T_{0}^{0}$ stands for the zero-zero component of the energymomentum tensor, which for the scalar field is derived from the action (2.8) and given by

$$
\begin{aligned}
T_{M}^{N}= & (1-2 \xi) \phi_{, M} \phi^{, N}+\frac{4 \xi-1}{2} \phi_{, L} \phi^{, L} \delta_{M}^{N}-2 \xi \phi_{; M}{ }^{; N} \phi \\
& +2 \xi \phi \square \phi \delta_{M}^{N}+\frac{\xi}{2}\left(2 R_{M}^{N}-R \delta_{M}^{N}\right) \phi^{2} .
\end{aligned}
$$

Note that the interaction part of the action does not contribute to the field energy-momentum tensor. It is particularly obvious in the case under consideration since for a point-like charge with the source (2.11) the Lagrangian density reads $\mathcal{L}_{\text {int }}=\sqrt{-g} \phi j=q \delta^{d-1}\left(x-x^{\prime}\right)$ and does not depend on the metric.

Making use the fact that the field and the metric are static we have

$$
\begin{aligned}
T_{0}^{0}= & -\frac{1-4 \xi}{2} \phi_{, \mu} \phi^{\mu}+2 \xi \phi \frac{1}{\sqrt{-g}} \partial_{\mu}\left[\sqrt{-g} g^{\mu \nu} \partial_{\nu} \phi\right] \\
& +\xi\left(R_{0}^{0}-\frac{1}{2} R\right) \phi^{2} .
\end{aligned}
$$

Substituting $T_{0}^{0}$, the scalar-field energy is given by

$$
\begin{aligned}
U_{\mathrm{sc}}= & \frac{1-4 \xi}{2} \int d^{d-1} x \partial_{\mu}\left(\sqrt{-g} g^{\mu v} \phi \partial_{\nu} \phi\right) \\
& -\frac{1}{2} \int d^{d-1} x\left[\phi \partial_{\mu}\left(\sqrt{-g} g^{\mu \nu} \partial_{\nu} \phi\right)\right. \\
& \left.+\sqrt{-g} \xi\left(2 R_{0}^{0}-R\right) \phi^{2}\right]
\end{aligned}
$$

and integrating with the help of the Gauss theorem, only the second integral survives. Simplifying and by the field equations (2.10), (2.12) becomes

$U_{\mathrm{sc}}=\frac{1}{2} \int d^{d-1} x \sqrt{-g}\left[\phi j-2 \xi R_{0}^{0} \phi^{2}\right]$.

The corresponding form via the Green function of Eq. (2.10) reads

$$
\begin{aligned}
U_{\mathrm{sc}}= & \frac{1}{2} \int d^{d-1} x d^{d-1} x^{\prime} \sqrt{g(x) g\left(x^{\prime}\right)} j(x) G\left(x, x^{\prime}\right) j\left(x^{\prime}\right) \\
& -\xi \int d^{d-1} x \sqrt{-g} R_{0}^{0} \phi^{2},
\end{aligned}
$$

where $G\left(x, x^{\prime}\right)$ satisfies

$$
\begin{aligned}
& \partial_{\mu}\left(\sqrt{-g} g^{\mu \nu} \partial_{\nu} G\left(x, x^{\prime}\right)\right)-\xi R \sqrt{-g} G\left(x, x^{\prime}\right) \\
& \quad=-\sqrt{-g} \delta^{d-1}\left(x, x^{\prime}\right) .
\end{aligned}
$$

Thus for a point charge localized at the point $x$ of the spacetime from Eq. (2.11) we get

$U_{\mathrm{sc}}(x)=\frac{1}{2} q^{2} G(x, x)-\xi \int d^{d-1} x \sqrt{-g} R_{0}^{0} \phi^{2}$.

Note that for the general case of a static spacetime one has $g=g_{00} \operatorname{det}\left(g_{\mu \nu}\right)$, while $R$ in Eq. (2.15) stands for the scalar curvature of the whole $d$-dimensional space.

In addition, if the spacetime is ultrastatic (i.e. $g_{00}=-1$ ), then $g=-\operatorname{det}\left(g_{\mu \nu}\right), R_{0}^{0}=0$, and (2.16) takes the form

$U_{\mathrm{sc}}(x)=\frac{1}{2} q^{2} G(x, x)$,

where $G$ is a solution of the equation

$$
\begin{aligned}
& \partial_{\mu}\left(\sqrt{\mathfrak{g}} g^{\mu \nu} \partial_{\nu} G\left(x, x^{\prime}\right)\right)-\xi \mathfrak{R} \sqrt{\mathfrak{g}} G\left(x, x^{\prime}\right) \\
& \quad=-\sqrt{\mathfrak{g}} \delta^{d-1}\left(x, x^{\prime}\right),
\end{aligned}
$$


where $\mathfrak{g}=\operatorname{det}\left(g_{\mu \nu}\right)$ and $\mathfrak{R}$ is the corresponding scalar curvature. That is, $G$ is the Green function on the $(d-1)$ dimensional space with the metric $g_{\mu \nu}$ with Euclidean signature and the curvature $\mathfrak{R}$.

Now let us suppose that there exists at least one flat extra spatial dimension, say $x_{d-1}$. Then formally identifying $i x_{d-1}=t$, one notices that Eq. (2.15) for the static Green function coincides with the full field equation (3.1) for the Euclidean Green function $G^{E}\left(x, x^{\prime} \mid d-1, n\right)$ in the spacetime with $(d-1)$ spacetime dimensions and $n$-dimensional conical subspace. Finally, with the use of the well-known relation between Euclidean $G^{E}$ and Feynman $G^{F}$ Green functions, we obtain, in this case,

$U_{\mathrm{sc}}=\frac{1}{2} q^{2} G^{E}(x, x \mid d-1, n)=-\frac{i}{2} q^{2} G^{F}(x, x \mid d-1, n)$.

One can study the self-energy of a static electric charge along the same lines.

In this case the solution of the Maxwell equations is static, with the $d$-potential $A_{M}=\left(A_{0}(x), 0, \ldots, 0\right)$ if the current equals $\left.J^{M}=(J(x), 0, \ldots, 0)\right)$. The only non-trivial component of the Maxwell equations is

$\partial_{\mu}\left(\sqrt{-g} g^{00} g^{\mu \nu} \partial_{\nu} A_{0}\right)=-\sqrt{-g} J$,

so for the electrostatic self-energy one obtains (e.g. see [40])

$$
\begin{aligned}
U_{\mathrm{el}} & =-\frac{1}{2} \int d^{d-1} x \sqrt{-g} A_{0} J \\
& =\frac{1}{2} \int d^{d-1} x \sqrt{-g} \int d^{d-1} x^{\prime} \sqrt{-g^{\prime}} J(x) G\left(x, x^{\prime}\right) J\left(x^{\prime}\right),
\end{aligned}
$$

where the Green function of Eq. (2.19) is defined as the solution of

$\partial_{\mu}\left(\sqrt{-g} g^{00} g^{\mu \nu} \partial_{\nu} G\left(x, x^{\prime}\right)\right)=\sqrt{-g} \delta^{d-1}\left(x, x^{\prime}\right)$.

So, for the point charge, when the charge density $J=$ $e \delta^{d-1}\left(x, x^{\prime}\right)$, we obtain

$U_{\mathrm{el}}=\frac{1}{2} e^{2} G(x, x)$

In the particular case of an ultrastatic space Eq. (2.21) takes the form

$\partial_{\mu}\left(\sqrt{\mathfrak{g}} g^{\mu \nu} \partial_{\nu} G\left(x, x^{\prime}\right)\right)=-\sqrt{\mathfrak{g}} \delta^{d-1}\left(x, x^{\prime}\right)$.
This equation coincides with Eq. (2.16) if $\xi=0$. Using this fact one finds that

$U_{\mathrm{el}}=\left.\frac{1}{2} e^{2} G^{E}(x, x \mid d-1, n)\right|_{\xi=0}$.

Consequently, on the background under consideration the electrostatic self-energy can be obtained from the scalar one if we put $\xi=0$ and replace $q^{2}$ by $e^{2}$.

The spacetime of interest here, see Eq. (2.5), i.e. the $d$ dimensional spacetime with $n$-dimensional subspace with a solid or planar angle deficit, satisfies the ultrastaticity condition, so we will use the simple formulas (2.18) and (2.23).

\section{Green function: perturbation theory}

For our background metric (2.5) the exact Green function is unknown. Taking into account the fact that $(1-\beta) \ll$ 1 we make use of the perturbation-theory techniques. The Feynman propagator for the scalar field in curved background satisfies the equation ${ }^{1}$

$\mathcal{L}(x, \partial) G^{F}\left(x, x^{\prime} \mid d, n\right)=-\delta^{d}\left(x-x^{\prime}\right)$,

where $\mathcal{L}(x, \partial)$ stands for the field-equation operator and determined by the background metric.

Following Schwinger [41], we rewrite Eq. (3.1), in the operator form

$\mathfrak{L} \mathcal{G}=-1, \quad \mathcal{G}=-\mathfrak{L}^{-1}$.

If the operator $\mathfrak{L}$ is allowed to be expressed as $\mathfrak{L}=\mathfrak{L}_{0}+$ $\delta \mathfrak{L}$, where $\delta \mathfrak{L}$ is considered as a small perturbation, then representing the solution of Eq. (3.2) in the form $\mathcal{G}=\mathcal{G}_{0}+\delta \mathcal{G}$, with $\mathcal{G}_{0}=-\mathfrak{L}_{0}{ }^{-1}$ being the unperturbed Green function, one obtains

$$
\begin{aligned}
\mathcal{G} & =\left[-\mathfrak{L}_{0}\left(1-\mathcal{G}_{0} \delta \mathfrak{L}\right)\right]^{-1} \\
& =\mathcal{G}_{0}+\mathcal{G}_{0} \delta \mathfrak{L} \mathcal{G}_{0}+\mathcal{G}_{0} \delta \mathfrak{L} \mathcal{G}_{0} \delta \mathfrak{L} \mathcal{G}_{0}+\cdots
\end{aligned}
$$

In the case under consideration $\mathfrak{L}_{0}$ is determined by the zeroth order in the small quantity $(1-\beta)$, hence

$$
\mathcal{L}_{0}(x, \partial)=\partial^{2}, \quad \partial^{2} \equiv \eta^{M N} \partial_{M} \partial_{N}
$$

The perturbation operator

$\delta \mathcal{L}(x, \partial)=\partial_{M}\left(\sqrt{-g} g^{M N} \partial_{N}\right)-\partial^{2}-\sqrt{-g} \xi R$

\footnotetext{
${ }^{1}$ In the following we define the Feynman propagator as $G^{F}\left(x, x^{\prime}\right)=$ $i\left\langle T\left[\phi(x) \phi\left(x^{\prime}\right)\right]\right\rangle_{\mathrm{vac}}$.
} 
to the first order in $(1-\beta)$ reads

$$
\begin{aligned}
\delta \mathcal{L}(x, \partial)= & n \alpha(r)\left(\partial_{0}^{2}-\sum_{N=n+1}^{d-1} \partial_{N}^{2}\right) \\
& -(n-2) \sum_{i=1}^{n}\left[\alpha(r) \partial_{i}^{2}+\left(\partial_{i} \alpha(r)\right) \partial_{i}\right]-\xi \gamma(r) .
\end{aligned}
$$

In order to compactify our equations below, let us introduce the notation

$\beta^{\prime} \equiv 1-\beta$

With the use of this notation

$\alpha(r)=\beta^{\prime} \ln r$

and

$\gamma(r)= \begin{cases}4 \pi \beta^{\prime} \delta^{2}(\mathbf{r}), & n=2 ; \\ 2(n-1)(n-2) \beta^{\prime} / r^{2}, & n \geqslant 3,\end{cases}$

with $\mathbf{r}=\left(x_{1}, x_{2}, \ldots, x_{n}\right)$.

In the problem at hand the function $G_{0}^{F}\left(x, x^{\prime}\right)=$ $\left\langle x\left|\mathcal{G}_{0}\right| x^{\prime}\right\rangle=-\left\langle x\left|\partial^{-2}\right| x^{\prime}\right\rangle$ in Fourier basis takes the form: ${ }^{2}$

$G_{0}^{F}\left(x-x^{\prime}\right)=\int \frac{d^{d} p}{(2 \pi)^{d}} \frac{\mathrm{e}^{i p\left(x-x^{\prime}\right)}}{p^{2}-i \varepsilon}$,

where $p^{2}=\mathbf{p}^{2}-\left(p^{0}\right)^{2}$ and $p x=\mathbf{p x}-p^{0} x^{0}$.

For the first-order correction to the Green function from (3.3) we get the following expression:

$$
\begin{aligned}
G_{1}^{F}\left(x, x^{\prime} \mid d, n\right)= & \left\langle x\left|\mathcal{G}_{0} \delta \mathfrak{L} \mathcal{G}_{0}\right| x^{\prime}\right\rangle \\
= & \int \frac{d^{d} q}{(2 \pi)^{d}} \mathrm{e}^{i q x} \int \frac{d^{d} p}{(2 \pi)^{d}} \mathrm{e}^{i p\left(x-x^{\prime}\right)} \\
& \times \frac{\delta \mathcal{L}(q, i p)}{\left[p^{2}-i \varepsilon\right]\left[(p+q)^{2}-i \varepsilon\right]}
\end{aligned}
$$

where $\delta \mathcal{L}^{(1)}(q, i p)$ is defined by

$\delta \mathcal{L}(q, i p)=\int d^{d} x \mathrm{e}^{-i q x}\left[\left.\delta \mathcal{L}(x, \partial)\right|_{\partial \rightarrow i p}\right]$

Here one implies that the differential operator $\delta \mathcal{L}(x, \partial)$ is prepared to the form where all differential operators stand before (at the right-hand side from) the coordinate functions,

\footnotetext{
${ }^{2}$ Hereafter the direct Fourier transform is defined as

$\mathcal{F}[\varphi(x)](q)=\int d^{d} x \varphi(x) \mathrm{e}^{-i q x}$,
}

and further one performs the substitution $\partial_{j} \rightarrow i p_{j}$ and calculates the Fourier transform, considering the $p_{j}$ as parameters.

In our problem the perturbation operator reads (3.5),

$\delta \mathcal{L}(q, i p)=\left[n p^{2}-2 \mathbf{p}^{2}+(n-2)(\mathbf{q p})\right] \mathcal{F}[\alpha](q)-\xi \mathcal{F}[\gamma](q)$,

where $\mathbf{p}=\left(p_{1}, \ldots, p_{n}\right)$ and $\mathbf{q}=\left(q_{1}, \ldots, q_{n}\right)$ are $n-$ dimensional conformal vectors with the Euclidean scalar product $(\mathbf{q p}) \equiv \delta_{i k} q^{i} p^{k}$, while $p^{2} \equiv \eta_{M N} p^{M} p^{N}$.

Making use of the explicit form of operator $\delta \mathcal{L}(x, \partial)$, substitution of (3.9) into Eq. (3.7) yields

$$
\begin{aligned}
G^{F}\left(x, x^{\prime} \mid d, n\right)= & G_{0}^{F}\left(x-x^{\prime}\right)+\int \frac{d^{d} q}{(2 \pi)^{d}} \mathrm{e}^{i q x} \int \frac{d^{d} p}{(2 \pi)^{d}} \\
& \times \frac{\mathrm{e}^{i p\left(x-x^{\prime}\right)}}{\left[p^{2}-i \varepsilon\right]\left[(p+q)^{2}-i \varepsilon\right]} \\
& \times\left[\left(n p^{2}-2 \mathbf{p}^{2}+(n-2)(\mathbf{q p})\right)\right. \\
& \times \mathcal{F}[\alpha](q)-\xi \mathcal{F}[\gamma](q)]
\end{aligned}
$$

Taking into account that formulas for the background curvature (3.6) differ for cases $n=2$ and $n \geqslant 3$, we consider here the generic case of a global monopole, while the case of a cosmic string is delegated to Sect. 7 below.

In this case (3.10) takes the form

$$
\begin{aligned}
G^{F} & \left(x, x^{\prime} \mid d, n\right) \\
= & G_{0}^{F}\left(x-x^{\prime}\right)-\frac{\Gamma(n / 2)}{2 \pi^{n / 2}} \int d^{n} q \frac{\mathrm{e}^{i \mathbf{q x}}}{\left(\mathbf{q}^{2}\right)^{n / 2}} \\
& \times \int \frac{d^{d} p}{(2 \pi)^{d}} \frac{\mathrm{e}^{i p\left(x-x^{\prime}\right)}}{\left[p^{2}-i \varepsilon\right]\left[(p+q)^{2}-i \varepsilon\right]} \\
& \times\left[n p^{2}-2 \mathbf{p}^{2}+(n-2)(\mathbf{q p})+2 \xi(n-1) \mathbf{q}^{2}\right],
\end{aligned}
$$

where we use the following well-defined Fourier transforms [42]:

$$
\begin{aligned}
\mathcal{F}[\ln r](q)= & -\frac{2^{d-1}}{\pi^{n / 2-d}} \frac{\Gamma(n / 2)}{\left(\mathbf{q}^{2}\right)^{n / 2}} \delta\left(q^{0}\right) \prod_{N=n+1}^{d-1} \delta\left(q^{N}\right), \\
\mathcal{F}\left[r^{-\lambda}\right](q)= & \frac{2^{d-\lambda}}{\pi^{n / 2-d}} \frac{\Gamma[(n-\lambda) / 2]}{\Gamma[\lambda / 2]} \\
& \times \frac{1}{\left(\mathbf{q}^{2}\right)^{(n-\lambda) / 2}} \delta\left(q^{0}\right) \prod_{N=n+1}^{d-1} \delta\left(q^{N}\right) .
\end{aligned}
$$


In Eq. (3.11) and in all subsequent equations $q \equiv$ $(0, \mathbf{q}, \underbrace{0,0 \ldots 0}_{d-n-1})$.

All the quantities we are interested in are expressed via the Feynman propagator $G^{F}\left(x, x^{\prime} \mid d, n\right)$ and its derivatives, evaluated in coincident points. The corresponding expressions diverge, and for their evaluation we make use of the dimensional-regularization method (see, e.g. [43]).

The dimensional regularization consists in the replacement of the determining function $G(x, x)$ by $G_{\text {reg }}(x, x)$, corresponding formally to the Green function in $D=(d-2 \varepsilon)$ dimensions. The subsequent renormalization includes the splitting of $G_{\text {reg }}(x, x)$ onto two parts; the first one diverges as $\varepsilon \rightarrow 0$, while the other is finite. The renormalization finishes with the neglect of the divergent part $G_{\text {div }}(x, x)$, with subsequent computation of the limit $\varepsilon \rightarrow 0$. But as it was remarked by Hawking [44], in the case of a curved space this procedure may be ambiguous, because in general there can be a variety of different ways of performing the analytic continuation from $d$ to $D$ dimensions. The simplest way is to take the product of the initial $d$-dimensional spacetime with a flat space with $D-d$ dimensions with subsequent analytic continuation with respect to the extra dimensions.

Fortunately, the spacetime of interest here has originally the structure demanded by this prescription. So, according to Hawking's prescription, we will define $G_{\text {ren }}^{F}(x, x \mid d, n)$ as a limit

$$
\begin{aligned}
& G_{\text {ren }}^{F}(x, x \mid d, n) \\
& \quad=\lim _{\varepsilon \rightarrow 0}\left[G_{\text {reg }}^{F}(x, x \mid D, n)-G_{\text {div }}^{F}(x, x \mid D, n)\right] .
\end{aligned}
$$

As it was shown by Hawking, results obtained by this prescription are in agreement with the ones obtained with the help of the method of generalized $\zeta$-functions.

\section{Renormalized $\left\langle\phi^{2}(x)\right\rangle$}

Now we proceed to a perturbative expression for the regularized value of vacuum averaged $\left\langle\phi^{2}(x)\right\rangle_{\text {ren }}$.

We define the Feynman propagator as $G^{F}\left(x, x^{\prime}\right)=$ $i\left\langle T\left[\phi(x) \phi\left(x^{\prime}\right)\right]\right\rangle_{\mathrm{vac}}$. So,

$\left\langle\varphi^{2}(x)\right\rangle_{\mathrm{ren}}=-i G_{\mathrm{ren}}^{F}(x, x \mid d, n)=G_{\mathrm{ren}}^{E}(x, x \mid d, n)$.

The first problem arising here is an expression arising in the zeroth order in $\beta^{\prime}$. Indeed, for the contribution from the first term on the right-hand side of (3.11) to the Green function taken in the limit of coincidence points, we have the formally divergent expression

$$
G_{0}^{F}(x, x)=\frac{1}{(2 \pi)^{d}} \int \frac{d^{d} p}{p^{2}} .
$$

However, all integrals of the form

$\int d^{d} p \frac{p_{i_{1}} \ldots p_{i_{k}}}{p^{2}}$

which diverge in UV- and/or in IR-limits and correspond to the "tadpole"-type diagrams in QFT, are set to have zero value (no tadpole prescription) within the dimensionalregularization technique (see, e.g., [45]). According to this prescription we shall put all terms of the form (4.2) equal to zero.

Thus, in the case $d \geqslant 4,3 \leqslant n \leqslant d-1$, and an arbitrary value of the coupling constant $\xi$, for the first non-vanishing contribution to the coincidence-point Green function one obtains from Eq. (3.11):

$$
\begin{aligned}
G^{F}(x, x \mid d, n)= & \beta^{\prime} \frac{\Gamma(n / 2)}{2 \pi^{n / 2}} \int d^{n} q \frac{\mathrm{e}^{i \mathbf{q} \mathbf{x}}}{\left(\mathbf{q}^{2}\right)^{n / 2}} \int \frac{d^{d} p}{(2 \pi)^{d}} \\
& \times \frac{2 \mathbf{p}^{2}-(n-2)(\mathbf{q p})-2 \xi(n-1) \mathbf{q}^{2}}{\left[p^{2}-i \varepsilon\right]\left[(p+q)^{2}-i \varepsilon\right]} .
\end{aligned}
$$

The integral over $d^{d} p$ diverges. However, it has a standard form for the QFT. Within the framework of the dimensional regularization one performs the Wick rotation

$p^{0} \rightarrow i p_{E}^{0}, \quad d^{d} p \rightarrow i d^{d} p_{E}$

and replaces the integral over $d^{d} p_{E}$ by the expression that formally corresponds to integration over a $(D-2 \varepsilon)$ dimensional $p_{E}$-space:

$\int \frac{d^{d} p}{(2 \pi)^{d}} \ldots \rightarrow i \mu^{2 \varepsilon} \int \frac{d^{D} p_{E}}{(2 \pi)^{D}} \cdots$

An arbitrary parameter $\mu$ with the dimension of reciprocal length is introduced to preserve the dimensionality of the regularized expression.

The computational technique for these integrals is well developed (e.g., see [45]) and we obtain ${ }^{3}$

$$
\begin{aligned}
& \int \frac{d^{D} p_{E}}{(2 \pi)^{D}} \frac{2 \mathbf{p}^{2}-(n-2)(\mathbf{q} \mathbf{p})-2 \xi(n-1) \mathbf{q}^{2}}{p_{E}^{2}(p+q)_{E}^{2}} \\
& =\left(1-\frac{\xi}{\xi_{D}}\right) \frac{2(n-1)}{(4 \pi)^{D / 2}} \frac{\Gamma^{2}(D / 2)}{\Gamma(D)} \frac{\Gamma(2-D / 2)}{\left(\mathbf{q}^{2}\right)^{1-D / 2}}
\end{aligned}
$$

where we have denoted

$\xi_{D} \equiv \frac{D-2}{4(D-1)}$

\footnotetext{
${ }^{3}$ For brief reference, we overview derivation of some of them in Appendix 1.
} 
Notice, when $\varepsilon=0$ and $\xi_{D}=\xi_{d}$, the field equation for a massless scalar field $\phi$ is invariant under conformal transformations of the metric.

For even $d$ Eq. (4.5) has a simple pole at $\varepsilon=0$, and under the removal of regularization the divergence in $G_{\text {reg }}^{E}(x, x \mid D, n)$ may arise due to this pole, or due to the $d^{n} q$-integration, or due to the two issues simultaneously.

Let consider this question in more detail.

Substituting (4.5) into (4.3) and making use of the integral (3.12) for the regularized vacuum mean $\left\langle\phi^{2}(x)\right\rangle$ we obtain (for all $3 \leqslant n \leqslant(d-1)$ ) the following expression:

$$
\begin{aligned}
\left\langle\phi^{2}(x)\right\rangle_{\mathrm{reg}}= & -i G_{\mathrm{reg}}^{F}(x, x \mid D, n) \\
= & \mu^{2 \varepsilon} \beta^{\prime} \frac{n-1}{4 \pi^{D / 2}} \frac{\Gamma(n / 2) \Gamma^{3}(D / 2)}{\Gamma(D)} \\
& \times\left(\frac{\xi}{\xi_{D}}-1\right) \frac{\Gamma\left(-\frac{D-2}{2}\right)}{\Gamma\left(-\frac{D-n-2}{2}\right)} \frac{1}{r^{D-2}} .
\end{aligned}
$$

We see that the behavior of the regularized $\operatorname{VEV}\left\langle\phi^{2}(x)\right\rangle_{\text {reg }}$ in the limit $\varepsilon \rightarrow 0$ is determined by the factor

$$
\Gamma\left(-\frac{D-2}{2}\right) / \Gamma\left(-\frac{D-n-2}{2}\right),
$$

and, therefore, it depends significantly upon the parity of the dimensionality both of the entire $d$-dimensional bulk and of its $n$-dimensional conical subspace.

Let us consider all possible cases.

- even $\boldsymbol{d}$, odd $\boldsymbol{n}$ In this case $(d-n-2) / 2$ is semi-integer, so Gamma-function in the denominator (4.7) takes a finite and non-zero value. The Gamma-function $\Gamma(1-D / 2)$ in the numerator of Eq. (4.7) has a simple pole in $\varepsilon=0$, thus when the regularization removed, the separation of divergent part may be performed with help of the Laurent expansion,

$$
\Gamma(-m+\varepsilon)=\frac{(-1)^{m}}{m !}\left(\frac{1}{\varepsilon}-\gamma+H_{m}+\mathcal{O}(\varepsilon)\right),
$$

where $\gamma$ is the Euler constant, and $H_{m}=\sum_{k=1}^{m} k^{-1}$ is the $m$ th harmonic number.

We obtain now

$$
\begin{aligned}
\left\langle\phi^{2}(x)\right\rangle_{\mathrm{div}}= & -i G_{\mathrm{div}}^{F}(x, x \mid d, n) \\
= & \frac{(-1)^{d / 2}}{\varepsilon} \frac{\beta^{\prime}}{2 \pi^{d / 2}} \frac{(n-1)}{(d-n)} \frac{\Gamma(n / 2) \Gamma^{2}(d / 2)}{\Gamma(d) \Gamma\left(-\frac{d-n}{2}\right)} \\
& \times\left(\frac{\xi}{\xi_{d}}-1\right) \frac{1}{r^{d-2}} .
\end{aligned}
$$

Notice, in the case of a conformal coupling $\left\langle\phi^{2}(x)\right\rangle_{\text {div }}$ vanishes.

Separation of the finite part of the regularized expression (4.6) is achieved by the following expansions:

$$
\begin{aligned}
& \frac{\xi}{\xi_{D}}-1=\left(\frac{\xi}{\xi_{d}}-1\right)+\varepsilon \frac{8 \xi}{(d-2)^{2}}+\mathcal{O}\left(\varepsilon^{2}\right), \\
& \frac{f(D) \mu^{2 \varepsilon}}{r^{D-2}}=\frac{f(d)}{r^{d-2}}\left[1+2 \varepsilon\left(\ln \mu r-\frac{f^{\prime}(d)}{f(d)}\right)+\mathcal{O}\left(\varepsilon^{2}\right)\right]
\end{aligned}
$$

where

$$
f(z) \equiv \frac{\Gamma^{3}(z / 2)}{\pi^{z / 2} \Gamma(z) \Gamma\left(\frac{2+n-z}{2}\right)},
$$

and that leads to the final result,

$$
\begin{aligned}
\left\langle\phi^{2}(x)\right\rangle_{\mathrm{ren}}= & (-1)^{(n-1) / 2} \beta^{\prime} \frac{(n-1) \Gamma(n / 2) \Gamma\left(\frac{d-n}{2}\right)}{2 \pi^{d / 2+1}} \frac{\Gamma^{2}(d / 2)}{\Gamma(d)} \\
& \times\left[\left(\frac{\xi}{\xi_{d}}-1\right) \ln \tilde{\mu} r+\frac{1}{(d-1)(d-2)}\right] \frac{1}{r^{d-2}} .
\end{aligned}
$$

Here and below the constant $\tilde{\mu}$ stands for the corresponding renormalized value of the constant $\mu$. In the expression above

$\tilde{\mu}=\mu \exp \left(-\frac{f^{\prime}(d)}{f(d)}+\frac{H_{d / 2-1}-\gamma}{2}+\frac{1}{(d-1)(d-2)}\right)$.

Notice, with the conformal coupling, the logarithmic term and the uncertainty related with the arbitrary constant $\tilde{\mu}$ in it disappear from $\left\langle\phi^{2}(x)\right\rangle_{\text {ren }}$.

Separately, we consider the case of higher-dimensional monopole, where $n=d-1$. Then from Eq. (4.9), making use of the well-known formulas for the Gamma-function,

$\Gamma(x) \Gamma(1-x)=\frac{\pi}{\sin \pi x}, \quad \Gamma(2 x)=\frac{2^{2 x-1} \Gamma(x) \Gamma(x+1 / 2)}{\sqrt{\pi}}$,

we have

$$
\begin{aligned}
\left\langle\phi^{2}(x)\right\rangle_{\mathrm{ren}}= & (-1)^{d / 2-1} \frac{\beta^{\prime}(d-2) \Gamma(d / 2)}{2^{d-1} \pi^{d / 2}(d-1)} \\
& \times\left[\left(\frac{\xi}{\xi_{d}}-1\right) \ln \tilde{\mu} r+\frac{1}{(d-1)(d-2)}\right] \frac{1}{r^{d-2}} .
\end{aligned}
$$


In particular, for the spacetime types $(4,3)$ and $(6,5)$ one obtains

$$
\begin{aligned}
& \left\langle\phi^{2}(x)\right\rangle_{\mathrm{ren}}=-\frac{\beta^{\prime}}{12 \pi^{2} r^{2}}\left[(6 \xi-1) \ln \tilde{\mu} r+\frac{1}{6}\right], \quad d=4 ; \\
& \left\langle\phi^{2}(x)\right\rangle_{\mathrm{ren}}=\frac{\beta^{\prime}}{20 \pi^{3} r^{4}}\left[(5 \xi-1) \ln \tilde{\mu} r+\frac{1}{20}\right], \quad d=6,
\end{aligned}
$$

which coincides (with the accuracy required) with the results of $[4,6,7]$, respectively. ${ }^{4}$

Finally, the renormalized VEV of $\left\langle\phi^{2}(x)\right\rangle$ for 3-dim monopole in six dimensions reads

$$
\begin{gathered}
\left\langle\phi^{2}(x)\right\rangle_{\mathrm{ren}}=-\frac{\beta^{\prime}}{120 \pi^{3} r^{4}}\left[(5 \xi-1) \ln \tilde{\mu} r+\frac{1}{20}\right], \\
d=6, n=3 .
\end{gathered}
$$

Thus the $(6,3)$-result is proportional to the $(6,5)$-one, as follows from (4.9). This expression differs from [5, Eq. 40] by the sign of the logarithmic term.

- odd $\boldsymbol{d}$ and $\boldsymbol{n}$ Here as $\varepsilon \rightarrow 0$ the Gamma-function $\Gamma(1-D / 2)$ in the numerator is finite, while $\Gamma(1-(D-$ $n) / 2$ ) in the denominator of (4.6) has a simple pole. Therefore, in the lowest order in $\beta^{\prime}\left\langle\phi^{2}(x)\right\rangle_{\text {ren }}$ vanishes. $^{5}$

- odd $\boldsymbol{d}$, even $\boldsymbol{n}$ In this case the two Gamma-functions, $\Gamma(1-D / 2)$ and $\Gamma(1-(D-n) / 2)$ in (4.7), are finite, hence $\left\langle\phi^{2}(x)\right\rangle_{\text {div }}=0$ and after some algebra (4.10) we arrive at

$$
\begin{aligned}
\left\langle\phi^{2}(x)\right\rangle_{\text {ren }}= & (-1)^{n / 2} \frac{\beta^{\prime}}{4 \pi^{d / 2}} \frac{(n-1) \Gamma(n / 2) \Gamma^{2}(d / 2)}{\Gamma(d)} \\
& \times \Gamma\left(\frac{d-n}{2}\right)\left(\frac{\xi}{\xi_{d}}-1\right) \frac{1}{r^{d-2}} .
\end{aligned}
$$

\footnotetext{
${ }^{4}$ Our result (4.12) coincides with the one of [4] numerically, since in the cited work it is the numerical computation that was done for several introduced (within their computational scheme) integrals (namely, [4, eqns. $(2.18,2.19)])$. However, these integrals may be computed analytically; doing this, the result coincides with our result (to the leading order in $\beta^{\prime}$ we are interested in here).

5 Unfortunately, we can say nothing about the result in the second order: neither whether it also vanishes, or if it has a finite value. Probably, the non-perturbative approach in some particular case $(d, n)$ of this type may shed light on this problem. The investigation of these effects lies beyond the mainline of our work here and hopefully will be considered later. The same concerns the cases with $\xi=\xi_{d}$ in Eqs. $(4.16,4.18)$, where the first-order value vanishes.
}

Hence for the $d$-dimensional monopole, $(d, d-1)$ spacetime, we have

$$
\begin{aligned}
& \left\langle\phi^{2}(x)\right\rangle_{\text {ren }} \\
& =(-1)^{n / 2} \beta^{\prime} \frac{(d-2) \Gamma(d / 2)}{2^{d} \pi^{d / 2-1}(d-1) r^{d-2}}\left(\frac{\xi}{\xi_{d}}-1\right) .
\end{aligned}
$$

In particular, for the five-dimensional monopole $(d=5$, $n=4)$ the $\operatorname{VEV}\left\langle\phi^{2}(x)\right\rangle_{\text {ren }}$ takes the form

$\left\langle\phi^{2}(x)\right\rangle_{\mathrm{ren}}=\beta^{\prime} \frac{3(16 \xi-3)}{2^{9} \pi r^{3}}$,

which coincides with the results of Refs. [5-7].

- even $\boldsymbol{d}$ and $\boldsymbol{n}$ Here the simple pole of $\Gamma(1-D / 2)$ in the numerator (4.7) is compensated by the one of the Gamma-function $\Gamma(-(D-n) / 2)$ in the denominator. The result of (4.7) at $\varepsilon=0$, thereby, equals the ratio of the corresponding residuals. Moreover, as in the previous case, the divergent part $\left\langle\phi^{2}(x)\right\rangle_{\text {div }}$ vanishes, and the VEV equals

$$
\begin{aligned}
& \left\langle\phi^{2}(x)\right\rangle_{\text {ren }} \\
& =(-1)^{n / 2} \beta^{\prime} \frac{(n-1) \Gamma\left(\frac{d-n}{2}\right)}{4 \pi^{d / 2}} \frac{\Gamma^{2}(d / 2) \Gamma(n / 2)}{\Gamma(d)} \\
& \quad \times\left(\frac{\xi}{\xi_{d}}-1\right) \frac{1}{r^{d-2}},
\end{aligned}
$$

and thus $\left\langle\phi^{2}(x)\right\rangle_{\text {ren }}$ vanishes (in lowest order in $\beta^{\prime}$ ) for the case of the conformal scalar field.

A direct comparison of (4.15) with Eq. (4.18) shows that within the accuracy of interest the two cases with even conical subdimensionality $n$ can be combined into a unified one, despite the intermediate formulas having been based on the drastically different behavior of the Gamma-function. However, for odd $n$ the result depends significantly upon the parity of the bulk's dimensionality.

Summarizing, in this section we have computed the renormalized vacuum averaged $\left\langle\phi^{2}\right\rangle_{\text {ren }}$ for a massless scalar field on the generalized background (2.5). We have made the computation up to the first order in $\beta^{\prime}$, but for arbitrary values of the coupling constant $\xi$ and for any dimension of the space $d \geqslant 4$ and any dimension of its conical subspace in the interval $3 \leqslant n \leqslant d-1$. For doing so we have used a perturbation technique combined with the method of dimensional regularization. For the case with even $d$ and odd $n$ (in particular, for the four-dimensional global monopole) it is the logarithmic factor $\ln \mu r$ that has the crucial significance for the field with nonconformal factor, since all finite non-logarithmic terms may be absorbed by the finite renormalization of $\mu$. 
The methods presented in this section may be used to compute the renormalized mean value of the energy-momentum tensor in a similar way.

\section{Renormalized energy-momentum tensor}

The total energy-momentum tensor derived from the action (2.8) is given by (2.13). In terms of the Green function, the regularized VEV of the energy-momentum tensor is given by

$$
\left\langle T_{M N}(x)\right\rangle_{\text {reg }}=-i \lim _{x^{\prime} \rightarrow x} D_{M N} G_{\text {reg }}^{F}\left(x, x^{\prime}\right),
$$

where $D_{M N}$ stands for the appropriate differential operator $\left(\nabla^{M}\right.$ and $\nabla^{M^{\prime}}$ denote the covariant derivative over $x^{M}$ and $x^{\prime M}$, respectively):

$$
\begin{aligned}
D_{M N}= & (1-2 \xi) \nabla_{M} \nabla_{N^{\prime}}+\frac{1}{2}(4 \xi-1) \nabla_{L} \nabla^{L^{\prime}} g_{M N} \\
& +\xi\left[R_{M N}-\frac{1}{2} R g_{M N}+2 \nabla_{L} \nabla^{L} g_{M N}-2 \nabla_{M} \nabla_{N}\right] .
\end{aligned}
$$

Taking into account the special significance of the minimally coupled field, and in order to dilute routine computations, it is natural to compute the renormalized vacuum momentum density separately for different powers of $\xi$. We start to separate $\xi$-terms already from definition: thereby we can split the energy-momentum tensor thus:

$T_{M N}=T_{M N}^{(0)}+\xi T_{M N}^{(\xi)}$

with

$$
\begin{aligned}
T_{M N}^{(0)}= & \phi, M \phi, N-\frac{1}{2} g_{M N} \phi, L \phi \\
T_{M N}^{(\xi)}= & -2 \phi, M \phi, N+2 \phi, L \phi^{, L} g_{M N}-2 \phi ; M N \phi \\
& +2 \phi \square \phi g_{M N}+\frac{1}{2}\left(2 R_{M N}-R g_{M N}\right) \phi^{2} .
\end{aligned}
$$

Each term here contains a quadratic form on $\phi$ and, therefore, can be derived from the Feynman propagator. Hence we may apply our point-splitting procedure for the derivatives combined with the perturbation-theory scheme, to reveal the contributions linear in $\beta^{\prime}$.

$T_{M N}^{(\xi)}$ contains the second covariant derivatives; computing them, one needs in the corresponding Christoffel symbols. In the coordinates specified, all non-vanishing Christoffel symbols are of order $\mathcal{O}\left(\beta^{\prime}\right)$. Given that the zeroth order (in $\beta^{\prime}$ ) of the Green function vanishes in our scheme (the no-tadpole prescription), the retaining of the Christoffel-part contribution yields the order $\mathcal{O}\left(\beta^{\prime 2}\right)$, i.e. exceeds the necessary accuracy. Hence we can neglect these terms and consider the derivatives as "flat".
Repeating the steps to construct the Green function, the first-order operator correction $\delta \mathcal{L}(x, \partial)$ also can be split as $\delta \mathcal{L}(x, \partial)=\delta \mathcal{L}^{(0)}(x, \partial)+\xi \delta \mathcal{L}^{(\xi)}(x, \partial)$ with $^{6}$

$$
\begin{aligned}
\delta \mathcal{L}^{(0)}(x, \partial)= & -n \alpha(r) \partial_{\sigma} \partial^{\sigma}-(n-2) \\
& \times\left[\alpha(r) \partial_{i} \partial^{i}+\left(\partial_{i} \alpha(r)\right) \partial^{i}\right], \\
& \times \delta \mathcal{L}^{(\xi)}(x, \partial)=-R(r) .
\end{aligned}
$$

In the following, the energy-momentum $\mathrm{VEV}$ in the first nonvanishing order reads schematically

$T_{M N}={ }^{0} T_{M N}+{ }^{1} T_{M N} \xi+{ }^{2} T_{M N} \xi^{2}$,

where

$\begin{aligned}{ }^{0} T_{M N} & =T_{M N}^{(0)}\left[\delta \mathcal{L}^{(0)}\right], \\ { }^{1} T_{M N} & =T_{M N}^{(0)}\left[\delta \mathcal{L}^{(\xi)}\right]+T_{M N}^{(\xi)}\left[\delta \mathcal{L}^{(0)}\right], \\ { }^{2} T_{M N} & =T_{M N}^{(\xi)}\left[\delta \mathcal{L}^{(\xi)}\right] .\end{aligned}$

The non-vanishing components of the Ricci tensor in our coordinates are given by (2.6) and survive in the conical sector only. For this reason, we should neglect the curvature-term in the last term in (5.2) since it contributes as $\mathcal{O}\left(\beta^{\prime 2}\right)$. Furthermore, after the replacement of the covariant derivatives by simple ones, the d'Alembert operator in $g_{M N} \phi \square \phi$ adds the multiplier $p^{2}$ into the numerator of Fourier integral. Multiplying by $p^{-2}(p+q)^{-2}$, this leads to the single-propagator Fourier integral, which vanishes in our scheme. Thereby, we can neglect this term also and replace (5.2) by its effective expression:

$T_{M N}^{(\xi)}=-2 \phi_{, M} \phi_{, N}+2 \phi_{, L} \phi^{, L} g_{M N}-2 \phi_{, M N} \phi$

Performing the Fourier transforms in (5.3), the $\xi$-separation in $\delta \mathcal{L}(q, i p)$ reads effectively

$$
\begin{aligned}
\delta \mathcal{L}^{(0)}(q, i p)= & -2^{n-1} \pi^{n / 2}(2 \pi)^{D-n} \Gamma(n / 2) \\
& \times \delta^{D-n}\left(q^{\sigma}\right) \frac{-2 \mathbf{p}^{2}+(n-2) \mathbf{q} \mathbf{p}}{|\mathbf{q}|^{n}} \\
\delta \mathcal{L}^{(\xi)}(q, i p)= & -2^{n}(n-1) \pi^{n / 2}(2 \pi)^{D-n} \Gamma(n / 2) \\
& \times \delta^{D-n}\left(q^{\sigma}\right)|\mathbf{q}|^{-(n-2)},
\end{aligned}
$$

so the latter actually does not depend upon $p^{M}$.

\footnotetext{
6 Within this section the index $\sigma$ runs over all "flat" indices: $\sigma=$ $0, n+1, \ldots, d-1$.
} 
5.1 Computation of $\left\langle T_{M N}\right\rangle_{\text {ren }}$ with minimal coupling

Starting from (5.7) and proceeding along the same lines as for $\left\langle\phi^{2}\right\rangle$, we obtain

$$
\begin{aligned}
& \left\langle{ }^{0} T_{M N}(x)\right\rangle_{\text {reg }} \\
& =\beta^{\prime} \int \frac{d^{D} q d^{D} p}{(2 \pi)^{2 D}} \mathrm{e}^{i q x} \frac{\delta \mathcal{L}^{(0)}(q, i p)}{\left[p^{2}-i \varepsilon\right]\left[(p+q)^{2}-i \varepsilon\right]} \\
& \quad \times\left(p_{M} p_{N}+q_{M} p_{N}-\frac{1}{2} \eta_{M N} \mathbf{q p}\right) .
\end{aligned}
$$

After the integration with help of integrals of Appendix 1, $\left\langle{ }^{0} T_{M N}(x)\right\rangle_{\text {reg }}$ reads

$$
\begin{aligned}
\left\langle{ }^{0} T_{M N}(x)\right\rangle_{\mathrm{reg}}= & -\beta^{\prime} \frac{\Gamma(n / 2) \Gamma^{2}(D / 2) \Gamma\left(-\frac{D-2}{2}\right)}{2^{D+3} \pi^{(D+n) / 2}(D+1) \Gamma(D)} \\
& \times \int d^{n} \mathbf{q} \frac{\mathrm{e}^{i \mathbf{q x}}}{|\mathbf{q}|^{2+n-D}}\left[-A_{D} \tilde{q}_{M} \tilde{q}_{N}+(n-1)\right. \\
& \left.\times\left(D^{2}-2 D-2\right) \mathbf{q}^{2} \eta_{M N}+2 \mathbf{q}^{2} \tilde{\eta}_{M N}\right]
\end{aligned}
$$

with $A_{D} \equiv D(D-n)+(n-2)(D-2)(D+1)$. Hereafter the "tilded" quantity with indices means that it equals the corresponding tensor with no tilde for the conical-subspace index, and it vanishes in the opposite case.

Integrating the remaining Fourier expressions, one arrives at

$$
\begin{aligned}
\left\langle{ }^{0} T_{M N}(x)\right\rangle_{\text {reg }}= & \beta^{\prime} \frac{\Gamma(n / 2) \Gamma^{3}(D / 2) \Gamma\left(-\frac{D-2}{2}\right)}{2^{3} \pi^{D / 2}(D+1) \Gamma(D) \Gamma\left(-\frac{D-n}{2}\right)} \frac{1}{r^{D}} \\
& \times\left[\frac{A_{D}}{D-n}\left(D \frac{\tilde{x}_{M} \tilde{x}_{N}}{r^{2}}-\tilde{\eta}_{M N}\right)\right. \\
& \left.-(n-1)\left(D^{2}-2 D-2\right) \eta_{M N}-2 \tilde{\eta}_{M N}\right] .
\end{aligned}
$$

\subsection{Computation of $\boldsymbol{\xi}$-terms}

Starting with the effective Fourier transforms (5.7) and (5.8), for the ${ }^{1} T_{M N}$-contributions we have explicitly:

$$
\begin{aligned}
T_{M N}^{(0)}\left[\delta \mathcal{L}^{(\xi)}\right]= & \beta^{\prime} \int \frac{d^{D} q}{(2 \pi)^{D}} \mathrm{e}^{i q x} \delta \mathcal{L}^{(\xi)}(q) \\
& \times \int \frac{d^{D} p}{(2 \pi)^{D}} \frac{1}{\left[p^{2}-i \varepsilon\right]\left[(p+q)^{2}-i \varepsilon\right]} \\
& \times\left(p_{M} p_{N}+q_{M} p_{N}-\frac{1}{2} \eta_{M N} \mathbf{q p}\right)
\end{aligned}
$$

$$
\begin{aligned}
& T_{M N}^{(\xi)}\left[\delta \mathcal{L}^{(0)}\right]=2 \beta^{\prime} \int \frac{d^{D} q d^{D} p}{(2 \pi)^{2 D}} \mathrm{e}^{i q x} \\
& \quad \times \frac{\delta \mathcal{L}^{(0)}(q, i p)}{\left[p^{2}-i \varepsilon\right]\left[(p+q)^{2}-i \varepsilon\right]}\left(-q_{M} p_{N}+\eta_{M N} \mathbf{q p}\right) .
\end{aligned}
$$

Substituting (5.8) into (5.12) and integrating over $p$ and $q^{\sigma}$, we obtain

$$
\begin{aligned}
T_{M N}^{(0)}\left[\delta \mathcal{L}^{(\xi)}\right] & \\
= & -\beta^{\prime} \frac{(n-1)(D-2) \Gamma(n / 2) \Gamma^{2}(D / 2) \Gamma\left(-\frac{D-2}{2}\right)}{2^{D+1} \pi^{(D+n) / 2} \Gamma(D)} \\
& \times \int d^{n} \mathbf{q} \frac{\mathrm{e}^{i \mathbf{q} \mathbf{x}}}{|\mathbf{q}|^{2+n-D}}\left(\tilde{q}_{M} \tilde{q}_{N}-|\mathbf{q}|^{2} \eta_{M N}\right) .
\end{aligned}
$$

Substituting (5.7) into (5.13) and integrating over $p$ and $q^{\sigma}$, one concludes

$$
T_{M N}^{(\xi)}\left[\delta \mathcal{L}^{(0)}\right]=T_{M N}^{(0)}\left[\delta \mathcal{L}^{(\xi)}\right]
$$

Thus combining (5.14) with (5.15) and integrating, for the regularized ${ }^{1} T_{M N}$ we arrive at

$$
\begin{aligned}
\left\langle{ }^{1} T_{M N}\right\rangle_{\mathrm{reg}}= & \beta^{\prime} \frac{(n-1)(D-2) \Gamma(n / 2) \Gamma^{3}(D / 2) \Gamma\left(-\frac{D-2}{2}\right)}{\pi^{D / 2} \Gamma(D) \Gamma\left(-\frac{D-n}{2}\right)} \\
& \times\left[\frac{1}{D-n}\left(\tilde{\eta}_{M N}-D \frac{\tilde{x}_{M} \tilde{x}_{N}}{r^{2}}\right)+\eta_{M N}\right] \frac{1}{r^{D}} .
\end{aligned}
$$

Computation of $\xi^{2}$-term. The term of interest here is given by

$$
\begin{aligned}
\left\langle T_{M N}^{(\xi)}\left[\delta \mathcal{L}^{(\xi)}\right]\right\rangle= & 2 \beta^{\prime} \int \frac{d^{D} q d^{D} p}{(2 \pi)^{2 D}} \mathrm{e}^{i q x} \\
& \times \frac{\delta \mathcal{L}^{(\xi)}(q, i p)}{\left[p^{2}-i \varepsilon\right]\left[(p+q)^{2}-i \varepsilon\right]} \\
& \times\left(q_{M} q_{N}+q_{M} p_{N}+\eta_{M N} \mathbf{q p}\right) .
\end{aligned}
$$

Integrating and substituting it with (5.8) into (5.6), we obtain

$$
\begin{aligned}
\left\langle T_{M N}^{(\xi)}\left[\delta \mathcal{L}^{(\xi)}\right]\right)_{\mathrm{reg}} \\
=-\beta^{\prime} \frac{(n-1)(D-1) \Gamma(n / 2) \Gamma^{2}(D / 2) \Gamma\left(-\frac{D-2}{2}\right)}{2^{D-1} \pi^{(D+n) / 2} \Gamma(D)} \\
\quad \times \int d^{n} \mathbf{q} \frac{\mathrm{e}^{i \mathbf{q x}}}{|\mathbf{q}|^{2+n-D}}\left(|\mathbf{q}|^{2} \eta_{M N}-\tilde{q}_{M} \tilde{q}_{N}\right)
\end{aligned}
$$

Comparing it with (5.14) and taking into account (5.15), one concludes

$$
\left\langle{ }^{2} T_{M N}\right\rangle_{\mathrm{reg}}=-\frac{2(D-1)}{D-2}\left\langle{ }^{1} T_{M N}\right\rangle_{\mathrm{reg}}=-\frac{1}{2 \xi_{D}}\left\langle{ }^{1} T_{M N}\right\rangle_{\mathrm{reg}} .
$$


Therefore their ratio does not depend on the conical subdimensionality $n$.

Integrating the last Fourier integral, we arrive at

$$
\begin{aligned}
& \left\langle{ }^{2} T_{M N}\right\rangle_{\mathrm{reg}} \\
& =-\beta^{\prime} \frac{2(n-1)(D-1) \Gamma(n / 2) \Gamma^{3}(D / 2) \Gamma\left(-\frac{D-2}{2}\right)}{\pi^{D / 2} \Gamma(D) \Gamma\left(-\frac{D-n}{2}\right)} \\
& \quad \times\left[\frac{1}{D-n}\left(\tilde{\eta}_{M N}-D \frac{\tilde{x}_{M} \tilde{x}_{N}}{r^{2}}\right)+\eta_{M N}\right] \frac{1}{r^{D}},
\end{aligned}
$$

therefore the combined regularized contribution of the $\xi$ terms equals

$$
\begin{aligned}
\left\langle T_{M N}\right. & \left.-{ }^{0} T_{M N}\right\rangle_{\mathrm{reg}} \\
= & \beta^{\prime} \frac{(D-2) \Gamma(n / 2) \Gamma^{3}(D / 2) \Gamma\left(-\frac{D-2}{2}\right)}{(n-1)^{-1} \pi^{D / 2} \Gamma(D) \Gamma\left(-\frac{D-n}{2}\right) r^{D}} \\
& \times\left[\frac{1}{D-n}\left(\tilde{\eta}_{M N}-D \frac{\tilde{x}_{M} \tilde{x}_{N}}{r^{2}}\right)+\eta_{M N}\right] \xi \\
& \times\left(1-\frac{\xi}{2 \xi_{D}}\right) .
\end{aligned}
$$

\subsection{Summary}

Combining (5.11) and (5.20), we obtain for the regularized value of energy-momentum VEV

$$
\begin{aligned}
& \left\langle T_{M N}\right\rangle_{\mathrm{reg}} \\
& =\frac{C \mu^{2 \varepsilon} \beta^{\prime}}{r^{D}}\left[\left(\frac{8(D-1)(n-1)}{D-n}\left(\xi-\xi_{D}\right)^{2}+\frac{1}{D^{2}-1}\right)\right. \\
& \quad \times\left(D \frac{\tilde{x}_{M} \tilde{x}_{N}}{r^{2}}-\tilde{\eta}_{M N}-(D-n) \eta_{M N}\right) \\
& \left.\quad+\frac{\eta_{M N}-\tilde{\eta}_{M N}}{D+1}\right]
\end{aligned}
$$

with

$C=\frac{\Gamma(n / 2) \Gamma^{3}(D / 2) \Gamma\left(-\frac{D-2}{2}\right)}{4 \pi^{D / 2} \Gamma(D) \Gamma\left(-\frac{D-n}{2}\right)}$.

We see that the classification by parity is based on the factor $\Gamma\left(-\frac{D-2}{2}\right) / \Gamma\left(-\frac{D-n}{2}\right)$. Given that $d-n \geqslant 1$, the first pole of the $\Gamma$-function in the denominator occurs at $d=n+2$; we return exactly to the same dimensionality splitting as for $\left\langle\phi^{2}\right\rangle_{\text {reg. }}$.

Hereafter it is more useful to consider the non-vanishing components of $T_{M N}$ separately:

1. The regularized vacuum energy density $\left\langle T_{00}(x)\right\rangle_{\text {reg }}$ (as well as the flat-sector spatial diagonal components $\left.\left\langle T_{\alpha \alpha}(x)\right\rangle\right)$ :

$$
\begin{aligned}
\left\langle T_{00}(x)\right\rangle_{\text {reg }}= & \frac{\mu^{2 \varepsilon} C(n-1) \beta^{\prime}}{r^{D}} \\
& \times\left[8(D-1)\left(\xi-\xi_{D}\right)^{2}-\frac{1}{D^{2}-1}\right] \\
= & -\left\langle T_{\alpha \alpha}(x)\right\rangle_{\text {reg }} ;
\end{aligned}
$$

2. The conical-subspace components $\left\langle T_{i k}(x)\right\rangle_{\mathrm{reg}}$ :

$$
\begin{aligned}
& \left\langle T_{i k}(x)\right\rangle_{\text {reg }} \\
& =\frac{C \mu^{2 \varepsilon} \beta^{\prime}}{r^{D+2}}\left[\left(\frac{8(D-1)(n-1)}{D-n}\left(\xi-\xi_{D}\right)^{2}\right.\right. \\
& \left.\left.\quad+\frac{1}{D^{2}-1}\right)\left(D x_{i} x_{k}-(D-n+1) r^{2} \delta_{i k}\right)\right] .
\end{aligned}
$$

With respect to the parity of $D$ and $n$ one distinguishes the following cases:

- $\boldsymbol{d}$ even, $\boldsymbol{n}$ odd The regularization removal in (5.22) is obtained in analogy with $\left\langle\phi^{2}\right\rangle_{\text {reg }}$ : the pole of the Gammafunction $\Gamma\left(-\frac{D-2}{2}\right)$ in the numerator gives rise to the corresponding divergent part (as $\epsilon \rightarrow 0$ )

$$
\begin{aligned}
\left\langle T_{M N}(x)\right\rangle_{\mathrm{div}}= & \frac{(-1)^{d / 2+1} \Gamma(n / 2) \Gamma^{2}(d / 2)}{4 \pi^{d / 2} \Gamma(d) \Gamma\left(-\frac{d-n}{2}\right)} \frac{\beta^{\prime}}{r^{d}} \frac{1}{\epsilon} \Theta_{M N} \\
\Theta_{M N} \equiv & \left(\frac{8(d-1)(n-1)}{d-n}\left(\xi-\xi_{d}\right)^{2}+\frac{1}{d^{2}-1}\right) \\
& \times\left(d \frac{\tilde{x}_{M} \tilde{x}_{N}}{r^{2}}-\tilde{\eta}_{M N}-(d-n) \eta_{M N}\right) \\
& +\frac{1}{d+1}\left(\eta_{M N}-\tilde{\eta}_{M N}\right),
\end{aligned}
$$

and to finite logarithmic and non-logarithmic terms.

In order to reveal the finite part, we have to make the following observation: as we have seen in Sect. 4, the divergent part corresponding to the pole of a Gamma-function is accompanied by the logarithmic term in the finite part, and there is some arbitrariness in the non-logarithmic term, related with the finite renormalization of logarithmic scale factor. Here we renormalize the tensor quantity, but the Gamma-function $\Gamma\left(-\frac{D-2}{2}\right)$, which produces a pole, sits in the common factor $C$ in (5.21), while the tensor part is regular. Also taking into account that the finite logarithmic shift due to expansion of $C$ is also common for the whole tensor, we expand $C$ in $\epsilon$ independently of the tensor structure, thus we have the unified logarithmic scale factor $\tilde{\mu}$ for all components of $T_{M N}$, while the tensor part in (5.21) has to be expanded additionally. 
Thus for the renormalized tensor we write generically

$$
\begin{aligned}
\left\langle T_{M N}(x)\right\rangle_{\text {ren }}= & \frac{(-1)^{d / 2-1} \Gamma(n / 2) \Gamma^{2}(d / 2)}{4 \pi^{d / 2} \Gamma(d) \Gamma\left(-\frac{d-n}{2}\right)} \frac{\beta^{\prime}}{r^{d}} \\
& \times\left[2 \Theta_{M N} \ln \tilde{\mu} r+A_{M N}\right] .
\end{aligned}
$$

It also allows for the logarithmic-scale finite shift, but within the scalar transformation. In other words, for the scale change $\mu \rightarrow \mu^{\prime}$ there is an uniparametrical arbitrariness of $A_{M N}$ in generic form

$A_{M N}^{\prime}=A_{M N}+2 \Theta_{M N} \ln \frac{\mu}{\mu^{\prime}}$.

Expanding

$$
\begin{aligned}
& \frac{8(n-1)\left(\xi-\xi_{D}\right)^{2}}{(D-1)^{-1}(D-n)}+\frac{1}{D^{2}-1} \\
& =\left[\frac{8(n-1)\left(\xi-\xi_{d}\right)^{2}}{(d-1)^{-1}(d-n)}+\frac{1}{d^{2}-1}\right] \\
& \quad+\left[\left(\frac{4(n-1)\left(\xi-\xi_{n}\right)}{d-n}\right)^{2}-\frac{1}{(d+1)^{2}}\right] \varepsilon \\
& \quad+\mathcal{O}\left(\varepsilon^{2}\right)
\end{aligned}
$$

and fixing the logarithmic scale as before (as $\tilde{\mu}$, implying the absorption of all $D$-dependent coefficients in $C$ ) one obtains

$$
\begin{aligned}
A_{M N}= & \left((4 \xi-1)^{2}-\frac{1}{(d+1)^{2}}\right)(n-1) \eta_{M N} \\
& -\left[\left(\frac{4(n-1)\left(\xi-\xi_{n}\right)}{d-n}\right)^{2}+\frac{1}{(d+1)^{2}}\right] \tilde{\eta}_{M N} \\
& +\left[n\left(\frac{4(n-1)\left(\xi-\xi_{n}\right)}{d-n}\right)^{2}-(n-1)(4 \xi-1)^{2}\right. \\
& \left.+\frac{1}{(d+1)^{2}}\right] \frac{\tilde{x}_{M} \tilde{x}_{N}}{r^{2}} .
\end{aligned}
$$

For the renormalized vacuum energy density we obtain

$$
\begin{aligned}
\left\langle T_{00}(x)\right\rangle_{\text {ren }}= & \beta^{\prime} \frac{(-1)^{d / 2}(n-1) \Gamma(n / 2) \Gamma^{2}(d / 2)}{4 \pi^{d / 2} \Gamma(d) \Gamma\left(-\frac{d-n}{2}\right)} \\
& \times\left[\left(\frac{2}{d^{2}-1}-16(d-1)\left(\xi-\xi_{d}\right)^{2}\right)\right. \\
& \left.\times \ln \tilde{\mu} r+(4 \xi-1)^{2}-\frac{1}{(d+1)^{2}}\right] \frac{1}{r^{d}} .
\end{aligned}
$$

It is not hard to conclude that for the values of a curvature coupling

$$
\xi=\xi_{d} \pm \frac{1}{d-1} \sqrt{\frac{1}{8(d+1)}}
$$

the renormalized density $\left\langle T_{00}(x)\right\rangle_{\text {ren }}$ does not contain a logarithmic term and thereby does not depend upon the arbitrary constant $\tilde{\mu}$, while the divergent part vanishes: $\left\langle T_{00}(x)\right\rangle_{\text {div }}=$ 0 .

The renormalized $\left\langle T_{i k}(x)\right\rangle$ reads

$$
\begin{aligned}
& \left\langle T_{i k}(x)\right\rangle_{\text {ren }} \\
& =\beta^{\prime} \frac{(-1)^{d / 2-1} \Gamma(n / 2) \Gamma^{2}(d / 2)}{2 \pi^{d / 2} \Gamma(d) \Gamma\left(-\frac{d-n}{2}\right)} \\
& \quad \times\left[\left(\frac{8(d-1)(n-1)}{d-n}\left(\xi-\xi_{d}\right)^{2}+\frac{1}{d^{2}-1}\right)\right. \\
& \left.\quad \times\left(d x_{i} x_{k}-(d-n+1) r^{2} \delta_{i k}\right) \ln \tilde{\mu} r+\frac{r^{2}}{2} A_{i k}\right] \frac{1}{r^{d+2}} .
\end{aligned}
$$

In the case of spacetime $(4,3)$ Eq. $(5.25)$ reduces to

$$
\begin{aligned}
\left\langle T_{M N}\right\rangle_{\mathrm{ren}}= & \frac{\beta^{\prime}}{8 \pi^{2} r^{4}}\left[\left[( 8 ( \xi - \frac { 1 } { 6 } ) ^ { 2 } + \frac { 1 } { 9 0 } ) \left(4 \frac{\tilde{x}_{M} \tilde{x}_{N}}{r^{2}}\right.\right.\right. \\
& \left.\left.-\tilde{\eta}_{M N}-\eta_{M N}\right)+\frac{\eta_{M N}-\tilde{\eta}_{M N}}{30}\right] \\
& \left.\times \ln \tilde{\mu} r+\frac{1}{12} A_{M N}\right] .
\end{aligned}
$$

Furthermore, due to the (theoretical) arbitrariness of the constant $\tilde{\mu}$, the non-logarithmic $\xi^{2}$-terms may be absorbed by the logarithm, introducing the new constant $\tilde{\mu}^{\prime}$ :

$$
\begin{aligned}
\left\langle T_{00}(x)\right\rangle_{\text {ren }}= & \beta^{\prime} \frac{(-1)^{d / 2}(n-1) \Gamma(n / 2) \Gamma^{2}(d / 2)}{2 \pi^{d / 2} \Gamma(d) \Gamma\left(-\frac{d-n}{2}\right)} \\
& \times\left[\left(\frac{1}{d^{2}-1}-8(d-1)\left(\xi-\xi_{d}\right)^{2}\right) \ln \tilde{\mu}^{\prime} r\right. \\
& \left.-\frac{4 \xi}{d-1}+\frac{d^{3}-1}{\left(d^{2}-1\right)^{2}}\right] \frac{1}{r^{d}} .
\end{aligned}
$$

In accord with (5.26), this finite shift $\tilde{\mu} \rightarrow \tilde{\mu}^{\prime}=\mathrm{e}^{-1 /(d+1)} \tilde{\mu}$ generates the corresponding shift $A_{i k} \rightarrow A_{i k}^{\prime}$ of the conicalsector spatial components.

For the higher-dimensional hyper-monopole $(n=d-1)$ Eq. (5.25) reduces to

$$
\begin{aligned}
\left\langle T_{00}(x)\right\rangle_{\text {ren }}= & (-1)^{d / 2-1} \beta^{\prime} \frac{(d-2) \Gamma(d / 2)}{2^{d} \pi^{d / 2}(d-1)} \frac{1}{r^{d}} \\
& \times\left[\left(\frac{1}{d^{2}-1}-8(d-1)\left(\xi-\xi_{d}\right)^{2}\right) \ln \tilde{\mu}^{\prime} r\right. \\
& \left.-\frac{4 \xi}{d-1}+\frac{d^{3}-1}{\left(d^{2}-1\right)^{2}}\right] \\
\left\langle T_{i k}(x)\right\rangle_{\text {ren }}= & \beta^{\prime} \frac{(-1)^{d / 2} \Gamma(d / 2)}{(4 \pi)^{d / 2}(d-1)}
\end{aligned}
$$




$$
\begin{aligned}
& \times\left[\left(8(d-1)(d-2)\left(\xi-\xi_{d}\right)^{2}+\frac{1}{d^{2}-1}\right)\right. \\
& \left.\times\left(d x_{i} x_{k}-2 r^{2} \delta_{i k}\right) \ln \tilde{\mu}^{\prime} r+\frac{r^{2}}{2} A_{i k}^{\prime}\right] \frac{1}{r^{d+2}} .
\end{aligned}
$$

Therefore, in the most important particular case of the spacetime $(4,3)$ - type it is given by

$$
\begin{aligned}
& \left\langle T_{00}(x)\right\rangle_{\text {ren }} \\
& =\frac{\beta^{\prime}}{4 \pi^{2}}\left[\left(4\left(\xi-\frac{1}{6}\right)^{2}-\frac{1}{90}\right) \ln \mu^{\prime} r+\frac{2}{9}\left(\xi-\frac{21}{100}\right)\right] \frac{1}{r^{4}} \\
& \left\langle T_{i k}(x)\right\rangle_{\text {ren }}=\frac{\beta^{\prime}}{4 \pi^{2}}\left[\left(8\left(\xi-\frac{1}{6}\right)^{2}+\frac{1}{90}\right)\right. \\
& \left.\quad \times\left(2 x_{i} x_{k}-r^{2} \delta_{i k}\right) \ln \tilde{\mu}^{\prime} r+\frac{r^{2}}{24} A_{i k}^{\prime}\right] \frac{1}{r^{6}}
\end{aligned}
$$

Now we can compare our result (5.29) with the part of the corresponding expression, linear in $\beta^{\prime}$, in [4], applied to the spacetime at hand.

The logarithmic expression in [4] within our accuracy ${ }^{7}$ generically is given by

$$
\begin{aligned}
& \left\langle T_{M N}(x)\right\rangle_{\log } \\
& =\frac{1}{160 \pi^{2}}\left[\left(\frac{1}{3}-\frac{10}{3} \xi+10 \xi^{2}\right) R_{; M N}-\frac{1}{6} \square R_{M N}\right. \\
& \left.\quad+\left(-\frac{1}{4}+\frac{10}{3} \xi-10 \xi^{2}\right) g_{M N} \square R\right] \ln \mu r,
\end{aligned}
$$

while the non-logarithmic term is arbitrary. Substituting the Ricci tensor and Ricci-scalar (2.6), and making use of

$$
\begin{aligned}
R_{; M N} & =\frac{4\left(1-\beta^{2}\right)}{r^{4}}\left(4 \frac{\tilde{x}_{M} \tilde{x}_{N}}{r^{2}}-\tilde{\eta}_{M N}\right), \\
\square R_{M N} & =4\left(1-\beta^{2}\right) \frac{\tilde{x}_{M} \tilde{x}_{N}}{r^{6}},
\end{aligned}
$$

one concludes that Eq. (5.29) has a discrepancy with (5.31) by a factor of 2 , for all monomials $\eta_{M N}, \tilde{\eta}_{M N}$ and $\tilde{x}_{M} \tilde{x}_{N}$, respectively. Meanwhile, the corresponding expression for $\left\langle\phi^{2}\right\rangle$ perfectly matches. Such a discrepancy implies necessity of the re-derivation of the generic expression in Ref. [47] (actually referred to by [4]). Following their ideology, based on the de Witt-Schwinger kernel, we could fix some inaccuracy of these works. ${ }^{8}$ Thus we think that if we take into account the fixing coefficient, our result (5.29) coincides with

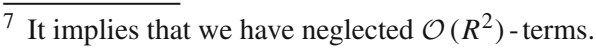

8 Actually, the pre-logarithmic expression (5.31), multiplied by 2, coincides with the pre-logarithmic coefficient in the logarithmically divergent part of the corresponding expression by Christensen [48] for the renormalized $\mathrm{VEV}$, but for massive scalar-field energy-momentum tensor.
}

the generic one (applied to the conical spacetime) in the logarithmic term, whereas it contains information as regards the non-logarithmic term.

In six dimensions $\left\langle T_{00}\right\rangle_{\text {ren }}$ disagrees with the leading terms of the perturbative expressions [6,7].

Conservation law and trace anomaly. The trace of the renormalized energy-momentum tensor (5.25) is determined by the traces of $\Theta_{M N}$ and $A_{M N}$, respectively. As it follows from (5.24), the trace of $\Theta_{M N}$ vanishes for the conformal field:

$\left.\operatorname{Sp} \Theta\right|_{\xi=\xi_{d}}=0$

hence the total trace is entirely determined by the one of $A_{M N}$ solely; computing it, one obtains

$$
\begin{aligned}
& \left\langle T_{M}^{M}\right\rangle_{\text {ren }} \\
& =\left.\frac{(-1)^{d / 2-1} \Gamma(n / 2) \Gamma^{2}(d / 2)}{4 \pi^{d / 2} \Gamma(d) \Gamma\left(-\frac{d-n}{2}\right)} \frac{\beta^{\prime}}{r^{d}} \operatorname{Sp} A\right|_{\xi=\xi_{d}} \\
& =\frac{(-1)^{(n+1) / 2}(n-1) \Gamma(n / 2) \Gamma^{2}(d / 2) \Gamma\left(\frac{d-n+2}{2}\right)}{2 \pi^{d / 2+1}\left(d^{2}-1\right) \Gamma(d)} \frac{\beta^{\prime}}{r^{d}} .
\end{aligned}
$$

For the known particular non-perturbative case $(4,3)$, the trace anomaly (5.32) equals (with our accuracy) its correct value [46]:

$$
\begin{aligned}
\left\langle T_{M}^{M}\right\rangle_{\text {ren }} & =\frac{1}{2880 \pi^{2}}\left[\square R-R_{M N} R^{M N}+R_{M N K L} R^{M N K L}\right] \\
& =\frac{1}{360} \frac{\beta^{\prime}}{r^{4}}+\mathcal{O}\left(\beta^{\prime 2}\right) .
\end{aligned}
$$

Such a coincidence also occurs in six dimensions [53] for the spacetimes $(6,5)$ and $(6,3)$.

In order to prove the conservation law, after some straightforward algebra 9 one can directly check the following two properties:

$\partial_{N}\left(\frac{\Theta^{M N}}{r^{d}}\right)=0, \quad \frac{2 \Theta^{M N} \tilde{x}_{N}}{r^{d+2}}+\partial_{N}\left(\frac{A^{M N}}{r^{d}}\right)=0$,

where we recall that first order implies the "common" derivatives. Thus the renormalized energy-momentum tensor is conserved.

- $\boldsymbol{d}$ and $\boldsymbol{n}$ odd Now $\Gamma\left(-\frac{D-2}{2}\right)$ in the numerator is regular, while $\Gamma\left(-\frac{D-n}{2}\right)$ in the denominator is infinite, hence the

\footnotetext{
9 A useful identity here is$$
2\left(\frac{8(d-1)(n-1)\left(\xi-\xi_{d}\right)^{2}}{(d-n)}+\frac{1}{d^{2}-1}\right)-\left(\frac{4(n-1)\left(\xi-\xi_{n}\right)}{\sqrt{d-n}}\right)^{2}
$$$$
=(n-1)(4 \xi-1)^{2}-\frac{1}{d+1} \text {. }
$$ 
total renormalized $\left\langle T_{M N}(x)\right\rangle$ vanishes:

$$
\left\langle T_{M N}(x)\right\rangle_{\text {ren }}=0
$$

in accord with the corresponding value of $\left\langle\phi^{2}\right\rangle .{ }^{10}$

- $\boldsymbol{d}$ odd, $\boldsymbol{n}$ even Here both $\Gamma\left(-\frac{D-2}{2}\right)$ in the numerator and $\Gamma\left(-\frac{D-n}{2}\right)$ in the denominator are regular, with semiinteger arguments, so $\left\langle T_{M N}(x)\right\rangle_{\mathrm{div}}=0$ and we have simply

$$
\begin{aligned}
& \left\langle T_{M N}(x)\right\rangle_{\text {ren }} \\
& =\frac{\Gamma(n / 2) \Gamma^{3}(d / 2) \Gamma\left(-\frac{d-2}{2}\right)}{4 \pi^{d / 2}\left(d^{2}-1\right) \Gamma(d) \Gamma\left(-\frac{d-n}{2}\right)} \frac{\beta^{\prime}}{r^{d}} \Theta_{M N} .
\end{aligned}
$$

Transforming it with the help of (4.10), one obtains

$$
\begin{aligned}
& \left\langle T_{M N}(x)\right\rangle_{\text {ren }} \\
& \quad=(-1)^{n / 2-1} \frac{\Gamma(n / 2) \Gamma^{2}(d / 2) \Gamma\left(\frac{d-n+2}{2}\right)}{4 \pi^{d / 2} \Gamma(d)} \frac{\beta^{\prime}}{r^{d}} \Theta_{M N} .
\end{aligned}
$$

In particular, for the $d$-dimensional monopole $(n=d-$ 1) the renormalized energy-momentum tensor reads

$$
\begin{aligned}
\left\langle T_{M N}\right\rangle_{\text {ren }}= & \frac{\pi^{1-d / 2} \Gamma(d / 2)}{(-4)^{(d+1) / 2}} \frac{\beta^{\prime}}{r^{d}}\left[\left(8(d-2)\left(\xi-\xi_{d}\right)^{2}\right.\right. \\
& \left.+\frac{d+1}{\left(d^{2}-1\right)^{2}}\right)\left(d \frac{\tilde{x}_{M} \tilde{x}_{N}}{r^{2}}-\tilde{\eta}_{M N}-\eta_{M N}\right) \\
& \left.+\frac{\eta_{M N}-\tilde{\eta}_{M N}}{d^{2}-1}\right] .
\end{aligned}
$$

- $\boldsymbol{d}$ and $\boldsymbol{n}$ even Here both $\Gamma\left(-\frac{D-2}{2}\right)$ in the numerator and $\Gamma\left(-\frac{D-n}{2}\right)$ in the denominator are singular, so their ratio is determined by the ratio of the corresponding residuals (4.8).

Thus $\left\langle T_{M N}(x)\right\rangle_{\mathrm{div}}=0$, and

$$
\begin{aligned}
& \left\langle T_{M N}(x)\right\rangle_{\text {ren }} \\
& \quad=(-1)^{n / 2-1} \frac{\Gamma(n / 2) \Gamma^{2}(d / 2) \Gamma\left(\frac{d-n+2}{2}\right)}{4 \pi^{d / 2} \Gamma(d)} \frac{\beta^{\prime}}{r^{d}} \Theta_{M N} .
\end{aligned}
$$

Again, Eqs. (5.35) and (5.36) are identical and represent the unified expression for even $n$, like for $\left\langle\phi^{2}\right\rangle$.

For the cases with no divergent part, the energy-momentum tensor is just proportional to $\Theta_{M N}$, hence the conservation law follows immediately: $\partial_{N}\left(r^{-d} \Theta^{M N}\right)=0$, while the trace anomaly to the first order disappears:

$\left.\left\langle T_{M}^{M}\right\rangle_{\text {ren }} \propto \Theta_{M}^{M}\right|_{\xi=\xi_{d}}=0$,

\footnotetext{
${ }^{10}$ See the footnote 5 on page 10 .
}

which for odd $d$ agrees with the non-perturbative result in literature.

Summarizing, in this section we have computed the renormalized vacuum averaged $\left\langle T_{M N}\right\rangle_{\text {ren }}$ of the massless scalar field in the background of (global) monopole up to the first order in $\beta^{\prime}$. Computing along the same ideology as in previous section, we obtain the same splitting with respect to the parity of the dimensionalities of the total spacetime and its deficit-angle submanifold. Here the most actual case with even $d$ and odd $n$ (in particular, for the (4,3)-type of a spacetime) requires higher accuracy when working with logarithms, due to the tensorial structure of $\left\langle T_{M N}\right\rangle_{\text {reg. }}$. The logarithmic mass-scale change generates the uniparametric equivalence class of the non-logarithmic symmetric tensors $A_{M N},{ }^{11}$ representing the linear shell of monomials $\eta_{M N}$, $\tilde{\eta}_{M N}$ and $\tilde{x}_{M} \tilde{x}_{N}$. For definite value of $\xi$, the logarithmic term and corresponding logarithmic uncertainty can be removed from $\left\langle T_{00}\right\rangle_{\text {ren }}$. However, contrary to the case of $T_{00}$, no value of the coupling $\xi$ kills the logarithmic term arising in $\left\langle T_{i k}\right\rangle_{\text {ren }}$, since both terms in the parentheses of (5.23) are positive. Finally, no value of $\xi$ eliminates the logarithmic arbitrariness both in $\left\langle\phi^{2}\right\rangle_{\text {ren }}$ and in $\left\langle T_{M N}\right\rangle_{\text {ren }}$ simultaneously. The computed energy-momentum tensor is conserved and provides the proper trace anomaly.

The other cases of $d$ and $n$ are similar to those of $\left\langle\phi^{2}\right\rangle_{\text {ren. }}$.

In the next section we show that the Green function obtained above enables one to consider the well-known purely classical problem of a gravity-induced self-action on a charge placed at a fixed point of the space under consideration.

\section{Static self-energy and self-force of a point-like charge}

As concluded in (2.18) and (2.23), the self-energy of a scalar $(q)$ or electric $(e)$ point charge in an ultrastatic $d$-dimensional spacetime is determined by the coincidence limit of the Euclidean Green function on the spacetime with the dimensionality $(d-1)$ :

$$
\begin{aligned}
& U_{\mathrm{sc}}(x)=\frac{q^{2}}{2} G_{\mathrm{ren}}^{E}(x, x \mid d-1, n), \\
& U_{\mathrm{el}}(x)=\left.\frac{e^{2}}{2} G_{\mathrm{ren}}^{E}(x, x \mid d-1, n)\right|_{\xi=0}
\end{aligned}
$$

Taking into account that for the self-energy the first nonvanishing order is $\mathcal{O}\left(\beta^{\prime}\right)$, one obtains to the lowest order simply

$\mathbf{F}_{\text {ren }}=-\operatorname{grad} U_{\text {ren }}$.

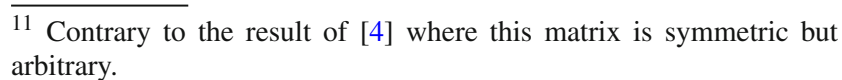


Moreover, a simple relation between scalar and electrostatic self-energy (2.23) enables one to restrict the consideration by the scalar one.

According to (4.6), the regularized scalar gravity-induced self-energy is given by

$$
\begin{aligned}
U_{\text {reg }}= & q^{2} \mu^{2 \varepsilon} \beta^{\prime} \frac{n-1}{8 \pi^{(D-1) / 2}} \frac{\Gamma(n / 2) \Gamma^{3}\left(\frac{D-1}{2}\right)}{\Gamma(D-1)} \\
& \times\left(\frac{\xi}{\xi_{D-1}}-1\right) \frac{\Gamma\left(-\frac{D-3}{2}\right)}{\Gamma\left(\frac{3-(D-n)}{2}\right)} \frac{1}{r^{D-3}} .
\end{aligned}
$$

Now the classification is determined basically by the factor

$$
\Gamma\left(-\frac{D-3}{2}\right) / \Gamma\left(\frac{3-D+n}{2}\right) .
$$

With respect to the parity of $d$ and $n$ one distinguishes the following cases:

- $\boldsymbol{d}$ even, $\boldsymbol{n}$ odd The Gamma-function $\Gamma\left(-\frac{D-3}{2}\right)$ is regular, while $\Gamma\left(\frac{3-D+n}{2}\right)$ tends to its pole (unless $D-n=1$ ). Therefore the renormalized self-energy and the self-force vanish generically in this case:

$$
U_{\text {ren }}=0, \quad \mathbf{F}_{\text {ren }}=0 .
$$

For the exceptional case $d-n=1$ both Gamma-functions are regular, hence

$$
\begin{aligned}
U_{\text {ren }}= & -q^{2} \beta^{\prime}(-1)^{d / 2} \frac{d-2}{8 \pi^{(d-3) / 2}} \frac{\Gamma^{3}\left(\frac{d-1}{2}\right)}{\Gamma(d-1)} \\
& \times\left(\frac{\xi}{\xi_{d-1}}-1\right) \frac{1}{r^{d-3}} .
\end{aligned}
$$

The corresponding self-force is given by

$$
\begin{aligned}
\mathbf{F}_{\text {ren }}= & -q^{2} \beta^{\prime}(-1)^{d / 2} \frac{(d-2)(d-3)}{8 \pi^{(d-3) / 2}} \frac{\Gamma^{3}\left(\frac{d-1}{2}\right)}{\Gamma(d-1)} \\
& \times\left(\frac{\xi}{\xi_{d-1}}-1\right) \frac{\mathbf{r}}{r^{d-1}} .
\end{aligned}
$$

Thus, at $\xi=\xi_{d-1}=(d-3) / 4(d-2)$ the renormalized self-energy and self-force vanish.

In the particular case of the $(4,3)$-spacetime one obtains

$U_{\text {ren }}=-q^{2} \beta^{\prime} \frac{\pi(8 \xi-1)}{2^{6} r}, \quad \mathbf{F}_{\text {ren }}=-q^{2} \beta^{\prime} \frac{\pi(8 \xi-1)}{2^{6}} \frac{\mathbf{r}}{r^{3}}$.

Thus, the point-like charge feels the monopole as a point charge with the magnitude $2^{-4} \beta^{\prime}(8 \xi-1) \pi^{2} q$ localized at the point $r=0$. For values $\xi>\xi_{3}=1 / 8$ the self-force is attractive (in particular, for the conformal coupling, $\xi=\xi_{4}=$ $1 / 6$ ), while for values $\xi<1 / 8$ the self-force is repulsive.

In the case of electrostatic self-action (according to Eq. (2.23) one has to put $\xi=0$ in (6.6) and replace $q^{2}$ by $e^{2}$ ) our result (6.6) coincides with the one of Ref. [50].

- $\boldsymbol{d}$ and $\boldsymbol{n}$ odd In this case the Gamma-function $\Gamma\left(-\frac{D-3}{2}\right)$ is singular, while $\Gamma\left(\frac{3-D+n}{2}\right)$ is regular. This leads to the non-zero diverging part, and the finite renormalized value of the self-energy takes the form

$$
\begin{aligned}
U_{\text {ren }}= & (-1)^{(n+3) / 2} q^{2} \beta^{\prime} \frac{n-1}{8 \pi(d+1) / 2} \\
& \times \frac{\Gamma(n / 2) \Gamma^{2}\left(\frac{d-1}{2}\right) \Gamma\left(\frac{d-n-1}{2}\right)}{\Gamma(d-1)} \\
& \times\left[\left(\frac{\xi}{\xi_{d-1}}-1\right) \ln \tilde{\mu} r+\frac{1}{(d-2)(d-3)}\right] \frac{1}{r^{d-3}}
\end{aligned}
$$

with arbitrary $\tilde{\mu} .^{12}$

The corresponding self-force reads

$$
\begin{aligned}
\mathbf{F}_{\text {ren }}= & (-1)^{(n+3) / 2} q^{2} \beta^{\prime} \frac{n-1}{8 \pi^{(d+1) / 2}} \frac{\Gamma(n / 2) \Gamma^{2}\left(\frac{d-1}{2}\right) \Gamma\left(\frac{d-n-1}{2}\right)}{\Gamma(d-1)} \\
& \times\left[\left(\frac{\xi}{\xi_{d-1}}-1\right)((d-3) \ln \tilde{\mu} r-1)+\frac{1}{(d-2)}\right] \frac{\mathbf{r}}{r^{d-1}} .
\end{aligned}
$$

For $\xi=\xi_{d-1}$ the result becomes free of uncertainty.

- $\boldsymbol{d}$ odd, $\boldsymbol{n}$ even Here the Gamma-function $\Gamma\left(-\frac{D-3}{2}\right)$ is singular, while $\Gamma\left(\frac{3-D+n}{2}\right)$ is also singular, unless $d=$ $n+1$. Hence, in the generic case the divergent part of the self-energy vanishes, and $U_{\text {ren }}$ is determined by the ratio of corresponding residuals:

$$
\begin{aligned}
U_{\text {ren }}= & (-1)^{n / 2} q^{2} \beta^{\prime} \frac{n-1}{8 \pi^{(d-1) / 2}} \frac{\Gamma(n / 2) \Gamma^{2}\left(\frac{d-1}{2}\right) \Gamma\left(\frac{d-n-1}{2}\right)}{\Gamma(d-1)} \\
& \times\left(\frac{\xi}{\xi_{d-1}}-1\right) \frac{1}{r^{d-3}} .
\end{aligned}
$$

The corresponding self-force equals

$$
\begin{aligned}
\mathbf{F}_{\text {ren }}= & (-1)^{n / 2} q^{2} \beta^{\prime} \frac{(n-1)(d-3)}{8 \pi^{(d-1) / 2}} \frac{\Gamma(n / 2) \Gamma^{2}\left(\frac{d-1}{2}\right) \Gamma\left(\frac{d-n-1}{2}\right)}{\Gamma(d-1)} \\
& \times\left(\frac{\xi}{\xi_{d-1}}-1\right) \frac{\mathbf{r}}{r^{d-1}} .
\end{aligned}
$$

$\overline{12}$ We remind the reader that throughout the main text we consider the case of a global monopole where $n \geqslant 3$ and thereby $d \geqslant 4$. The lowdimensional-gravity case $(3,2)$ will be considered in the next section. 
In the exceptional case of the higher-dimensional monopole $(d=n+1)$ the denominator $\Gamma\left(\frac{3-D+n}{2}\right)$ is regular, hence we return to the logarithmic case: along the same lines as previously we obtain

$$
\begin{aligned}
U_{\text {ren }}= & q^{2} \beta^{\prime} \frac{(-1)^{(d+1) / 2}}{8 \pi^{(d-1) / 2}} \frac{\Gamma^{2}\left(\frac{d-1}{2}\right)}{\Gamma(d-1)} \\
& \times\left[(d-2)\left(\frac{\xi}{\xi_{d-1}}-1\right) \ln \tilde{\mu} r+\frac{1}{d-3}\right] \frac{1}{r^{d-3}}, \\
\mathbf{F}_{\text {ren }}= & q^{2} \beta^{\prime} \frac{(-1)^{(d+1) / 2}}{8 \pi^{(d-1) / 2}} \frac{\Gamma^{2}\left(\frac{d-1}{2}\right)}{\Gamma(d-1)} \\
& \times\left[(d-2)\left(\frac{\xi}{\xi_{d-1}}-1\right)((d-3) \ln \tilde{\mu} r-1)+1\right] \frac{\mathbf{r}}{r^{d-1}} .
\end{aligned}
$$

- $\boldsymbol{d}$ and $\boldsymbol{n}$ even Here both Gamma-functions in (6.3) are regular, hence the divergent part vanishes and after transformations with the help of (4.10) we have just

$$
\begin{aligned}
U_{\text {ren }}= & (-1)^{n / 2} q^{2} \beta^{\prime} \frac{n-1}{8 \pi^{(d-1) / 2}} \frac{\Gamma(n / 2) \Gamma^{2}\left(\frac{d-1}{2}\right) \Gamma\left(\frac{d-n-1}{2}\right)}{\Gamma(d-1)} \\
& \times\left(\frac{\xi}{\xi_{d-1}}-1\right) \frac{1}{r^{d-3}}, \\
\mathbf{F}_{\text {ren }}= & (-1)^{n / 2} q^{2} \beta^{\prime} \frac{(n-1)(d-3)}{8 \pi^{(d-1) / 2}} \\
& \times \frac{\Gamma(n / 2) \Gamma^{2}\left(\frac{d-1}{2}\right) \Gamma\left(\frac{d-n-1}{2}\right)}{\Gamma(d-1)} \\
& \times\left(\frac{\xi}{\xi_{d-1}}-1\right) \frac{\mathbf{r}}{r^{d-1}} .
\end{aligned}
$$

To summarize based on the formal relation of the Feynman propagator with the Euclidean Green function in the coincidence-point limit, we have expressed the regularized self-action via the regularized Green function of the previous dimensionality. As before, the consideration splits into four characteristic cases of the parities $d$ and $n$, though here one meets the significant exceptions of the monopole background with no flat spatial dimensions $(n=d-1)$. In most cases the self-action looks like the flat-space Coulomb interaction of a charge $q$ with a charge $\propto\left(\xi-\xi_{d-1}\right) q$ placed into the monopole position, and vanishes for the particular value $\xi=\xi_{d-1}$ of the curvature coupling. In the case of odd $d$ while $n$ is odd or equal to $d-1$, there is an additional logarithmic multiplier, which depends on the arbitrary parameter $\tilde{\mu}$.

Finally, comparing (6.13) with (6.9) and (6.10), we notice that for even $n$ the cases with even and odd $d$ can be combined into the unified formula (except for the hyper-monopole case), in accord with the previous computations of the renormalized $\left\langle\phi^{2}\right\rangle$ and $\left\langle T_{M N}\right\rangle$.

\section{Vacuum polarization near cosmic string revisited}

Now consider the particular case of a two-dimensional $(n=$ 2 ) conical subspace. If $d=3(4)$ this space is the spacetime of a point mass (infinitely thin straight cosmic string).

This problem has been considered in a series of papers. The primary goal of our consideration is to show that there is some ambiguity in the computations [10-13,27] in the case of a non-minimally coupled massless scalar field.

Indeed, in the cited calculations the starting point is Eq. (5.1) with operator $D_{M N}$, whose form is determined by the classical expression for the energy-momentum tensor and thus includes the $\xi$-dependent terms. However, as the Green function of the spacetime at hand the Green function for a minimally coupled scalar field was used. Thus, it was supposed that one can extract a $\delta^{2}$-like potential from the wave equation, arguing it by the fact that the space is flat everywhere outside the point mass/cosmic string. This Green function does not depend on $\xi$ and in the limit $\beta \rightarrow 1$ tends to the flat Green function $G_{0}^{F}\left(x-x^{\prime}\right)$, which is the solution of the equation

$\eta^{M N} \partial_{M} \partial_{N} G_{0}^{F}\left(x-x^{\prime}\right)=-\delta^{d}\left(x-x^{\prime}\right)$.

On the other hand, we can start from the explicit equation

$\sqrt{-g}[\square-\xi R] G_{\xi}^{F}\left(x, x^{\prime}\right)=-\delta^{d}\left(x-x^{\prime}\right)$.

In the coordinates we use, the potential reads

$\gamma(r)=\sqrt{-g} \xi R=4 \pi \beta^{\prime} \xi \delta^{2}(\mathbf{r}), \quad \mathbf{r}=\left(x^{1}, x^{2}\right)$.

In Eq. (7.2) there are two independent parameters, namely $\beta^{\prime}$ and $\xi$. Suppose that there exists a limit of the Green function $G_{\xi}^{F}$, when

$\beta^{\prime} \rightarrow 0, \quad \xi \rightarrow \infty, \quad \lambda \equiv 4 \pi \xi \beta^{\prime}=$ const.

Let us denote it as $G_{\lambda}^{F}$. In this limit Eq. (7.2) takes the form

$\left[\eta^{M N} \partial_{M} \partial_{N}-\lambda \delta^{2}(\mathbf{x})\right] G_{\lambda}^{F}\left(x, x^{\prime}\right)=-\delta^{d}\left(x-x^{\prime}\right)$.

Thus the Aharonov-Bohm-like potential survives even in the case where the space is almost Euclidean. It is obvious that, if the limit does exist, $G_{\lambda}^{F}$ cannot be equal to the flat-space Green function $G_{0}^{F}$.

The corresponding equation for the scalar field $\phi$ can be reduced to a stationary two-dimensional Schrödinger-like equation with a planar $\delta^{2}$-function potential. Equations of this kind have been widely discussed in the literature. It was shown that these interactions require regularization and infinite renormalization of the coupling constant and lead to nontrivial physical results. Alternatively, one can follow a more satisfactory approach based on a self-adjoint extension of 
a noninteracting Hamiltonian, defined on a space with one extracted point (see [51,52] and the references therein).

We think that the example above demonstrates the necessity to revise the vacuum polarization effects on manifolds with $\delta^{2}$-like singularities. This problem demands consideration in more detail. Here we restrict ourselves by the consideration of this problem in the framework of the perturbation approach.

Thus, we start from Eq. (3.10) with the potential $\gamma$ defined by Eq. (7.3). The Fourier transform of this potential has the form

$$
\mathcal{F}[\gamma(r)]=4 \pi \beta^{\prime}(2 \pi)^{d-2} \delta\left(q^{0}\right) \prod_{N=3}^{d-1} \delta\left(q^{N}\right) .
$$

Substituting (7.5) into Eq. (3.10), we obtain, with our accuracy,

$$
\begin{aligned}
& G^{F}\left(x, x^{\prime} \mid d, 2\right) \\
& =G_{0}^{F}\left(x-x^{\prime}\right)+\frac{\beta^{\prime}}{\pi} \int d^{2} q \frac{\mathrm{e}^{i \mathbf{q} \mathbf{x}}}{\mathbf{q}^{2}} \\
& \quad \times \int \frac{d^{d} p}{(2 \pi)^{d}} \mathrm{e}^{i p\left(x-x^{\prime}\right)} \frac{\mathbf{p}^{2}-\xi \mathbf{q}^{2}}{\left[p^{2}-i \varepsilon\right]\left[(p+q)^{2}-i \varepsilon\right]}
\end{aligned}
$$

Starting from (7.6) and proceeding along the same line as in the previous sections, we obtain

$$
\begin{aligned}
\left\langle\phi^{2}(x)\right\rangle_{\mathrm{ren}} & =-i G_{\mathrm{ren}}^{F}(x, x \mid d, 2) \\
& =-\frac{\beta^{\prime}}{2 \pi^{d / 2}}\left(\frac{\xi}{\xi_{d}}-1\right) \frac{\Gamma^{3}(d / 2)}{(d-2) \Gamma(d)} \frac{1}{r^{d-2}}
\end{aligned}
$$

for the renormalized vacuum expectation value of the field square and

$$
\begin{aligned}
\left\langle T_{00}\right\rangle_{\text {ren }}= & \beta^{\prime} \frac{\Gamma^{3}(d / 2)}{4 \pi^{d / 2} \Gamma(d)}\left(8(d-1)\left(\xi-\xi_{d}\right)^{2}-\frac{1}{d^{2}-1}\right) \frac{1}{r^{d}} \\
\left\langle T_{11}\right\rangle_{\text {ren }}= & \beta^{\prime} \frac{\Gamma^{3}(d / 2)}{4 \pi^{d / 2} \Gamma(d)}\left[\frac{2\left(\xi-\xi_{d}\right)^{2}}{\xi_{d}}+\frac{1}{d^{2}-1}\right] \\
& \times\left(x_{1}^{2}-(d-1) x_{2}^{2}\right) \frac{1}{r^{d+2}}, \\
\left\langle T_{22}\right\rangle_{\text {ren }}= & \left.\left\langle T_{11}\right\rangle_{\text {ren }}\right|_{x_{1} \leftrightarrow x_{2}}, \\
\left\langle T_{12}\right\rangle_{\text {ren }}= & \left\langle T_{21}\right\rangle_{\text {ren }}=\beta^{\prime} \frac{d \Gamma^{3}(d / 2)}{4 \pi^{d / 2} \Gamma(d)} \\
& \times\left[\frac{2\left(\xi-\xi_{d}\right)^{2}}{\xi_{d}}+\frac{1}{d^{2}-1}\right] \frac{x_{1} x_{2}}{r^{d+2}}, \\
\left\langle T_{\alpha \beta}\right\rangle_{\text {ren }}= & -\delta_{\alpha \beta}\left\langle T_{00}\right\rangle_{\text {ren }}, \quad \alpha, \beta, \ldots=3, \ldots, d-1,
\end{aligned}
$$

for the non-zero components of $\left\langle T_{M N}\right\rangle_{\text {ren }}$.
The corresponding classical gravity-induced scalar selfenergy and self-force are given by

$$
\begin{aligned}
& U_{\text {ren }}=-q^{2} \beta^{\prime} \frac{\Gamma^{2}\left(\frac{d-1}{2}\right) \Gamma\left(\frac{d-3}{2}\right)}{8 \pi^{(d-1) / 2} \Gamma(d-1)}\left(\frac{\xi}{\xi_{d-1}}-1\right) \frac{1}{r^{d-3}}, \\
& \mathbf{F}_{\text {ren }}=-\frac{q^{2} \beta^{\prime}}{4 \pi^{(d-1) / 2}} \frac{\Gamma^{3}\left(\frac{d-1}{2}\right)}{\Gamma(d-1)}\left(\frac{\xi}{\xi_{d-1}}-1\right) \frac{\mathbf{r}}{r^{d-1}},
\end{aligned}
$$

unless $d=3$. Thus in any dimension the self-force is attractive for $\xi>\xi_{d-1}$, repulsive vice versa, and equal to zero if $\xi=\xi_{d-1}$. For all quantities discussed above, the infinite part of the regularized expression vanishes.

Self-action in (3,2)-spacetime. This particular case differs from the one considered above due to (6.2) diverges when $D \rightarrow 3$. Thus, we have to proceed the dimensionalregularization prescription and isolate the corresponding singular terms. The result is

$$
\begin{aligned}
& U_{\text {ren }}=-\frac{q^{2} \beta^{\prime}}{4 \pi}\left[\ln \tilde{\mu} r+2 \xi \ln ^{2} \tilde{\mu} r\right]+\text { const }, \\
& \mathbf{F}_{\text {ren }}=\frac{q^{2} \beta^{\prime}}{4 \pi}[1+4 \xi \ln \tilde{\mu} r] \frac{\mathbf{r}}{r^{2}} .
\end{aligned}
$$

The single-logarithmic term in $U_{\text {ren }}$ comes from a simple pole in the no- $\xi$-term and is similar to the $(d, d-1)$-type considered in (6.11). Contrary to (6.11), the squared-logarithmic term is obtained as a regularization of a second-order pole in $\xi$-term. ${ }^{13}$ For the minimal coupling (7.10) agrees with $[13,23,26,27]$.

To conclude, our results coincide with the ones of Refs. [10-14,23,25-27] in the case of a minimally coupled scalar field in the three-/four-dimensional spacetime, but they differ from those if $\xi \neq 0$. As mentioned above, this distinction is a consequence of the fact that the Green function satisfies Eq. (7.2). With respect to Eq. (7.1), the latter contains an additional two-dimensional $\delta^{2}(\mathbf{x})$-potential which was not taken into account in the cited references.

However, our result for $\left\langle T_{M N}\right\rangle_{\text {ren }}$ in four dimensions ${ }^{14}$ coincides with $[11,12]$ also for the particular value $\xi=\xi_{4}=$ $1 / 6$. This "occasional" coincidence follows from the fact that the sum $\left\langle T_{M N}^{(0)}\left[\delta \mathcal{L}^{(\xi)}\right]+T_{M N}^{(\xi)}\left[\delta \mathcal{L}^{(\xi)}\right]\right\rangle_{\text {reg }}$, representing the discrepancy, is proportional to $\xi\left(\xi-\xi_{D}\right)$. If the divergent part vanishes, which is the case for the cosmic string, then the latter equals $\xi\left(\xi-\xi_{d}\right)$ and thus vanishes for the conformal coupling also. Thus we expect that the coincidence for the conformal coupling occurs in the first computational order

\footnotetext{
13 Alternatively, it can be seen in the original $\xi$-coefficient (7.6) without regularization since after the Wick rotation it becomes the product of identical inverse Fourier integrals $\mathcal{F}^{-1}\left[q^{-2}\right]$ (3.12) well defined in the sense of distributions.

14 The same concerns the three-dimensional gravity-induced result [13] for $\xi=\xi_{3}$.
} 
(in angular-deficit series), while the difference is going to be visible in the next orders.

Notice that our results (7.7), (7.8), and (7.9) coincide with the $n \rightarrow 2$ limit of the results obtained in Sects. 4 and 5. First of all, as we mentioned, the monopole's results for even $n$ are combined (for odd and even $d$ ). Furthermore, we see that Eqs. (4.15), (4.18), (5.35), (5.36), (6.13), (6.10), and (6.13) are regular at $n=2$, though the initial expression for the Ricci scalar was singular in this limit (3.6), representing the only difference. Next, we observe that this difference disappears after the first Fourier transform. Indeed, the Fourier transform of the string's Ricci scalar perfectly coincides with the (regular) $n \rightarrow 2$ limit of the corresponding Fourier transform of the monopole's Ricci scalar, readily computed with help of (3.12). Since the rest of the computation is the same for a cosmic string and a global monopole, it is no wonder that we have obtained coincidence in the final formulas.

\section{Conclusion}

On curved backgrounds, being multidimensional generalizations of the well-known four-dimensional cosmic string/ global monopole, we have considered two at first glance disconnected problems - namely, the gravity-induced vacuum polarization of a massless scalar field and the classical selfaction of a static scalar or electric charge. However, the technique to solve the problems under consideration turned out to be similar, since it refers to getting a compact workable expression for the Green function and its derivatives in the coincidence-point limit for all $d \geqslant 3$ and $2 \leqslant n \leqslant d-1$, representing our primary particular goal. For this purpose we use the methods of perturbation theory. Taking into account the actual smallness of the angle deficit, we have performed computations in first order with respect to the angular-deficit value. Since, in principle, both the vacuum expectation values and the classical self-energy are divergent, for regularization and renormalization of these quantities we adapted the dimensional-regularization method.

The zeroth computational order is determined by the Minkowskian Green function and completely consists of the tadpole-like contributions (4.2). In quantum field theory, the appearance of divergences produced by tadpoles is explained by the fact that the perturbation theory is constructed with respect to a nonphysical vacuum, while their elimination is explained by the necessity of redefining the vacuum state. In the framework of the self-action, it is of interest to understand why similar divergences appear in the classical theory too. Following the prescriptions of quantum field theory, we assumed all expressions of the form (4.2) to be equal to zero. The motivation for this recipe is not associated in any way with quantum theory. Actually, it relies on the absence of dimensional parameters in the corresponding expression and, as a consequence, on the impossibility to assign some reasonable finite value, except zero, to such integrals under regularization. Therefore, this rule is also equally applicable within the classical field theory.

The desired effects are computed in the first order in $\beta^{\prime}$. Already starting from the Green function, for all of our computational tasks we meet the characteristic ratio of two Gamma-functions, which splits the consideration of all $(d, n)$-types onto four characteristic cases, depending on parities of $d$ and $n$. The poles of Gamma-function may arise in the numerator, in the denominator, or in both. However, in the very end of computation one can combine all formulas with even $n$ (for arbitrary $d$ ) into the unified case.

With the help of the regularized Green function we have computed the renormalized vacuum averaged $\left\langle\phi^{2}\right\rangle_{\text {ren }}$ and $\left\langle T_{M N}\right\rangle_{\text {ren }}$ for a massless scalar field coupled with the generalized conical background (2.5) via an arbitrary coupling $\xi$. The expressions for vacuum averaged $\left\langle\phi^{2}\right\rangle_{\text {ren }}$, corresponding to all characteristic cases (with our accuracy), vanish at $\xi=\xi_{d}$. In the case with even $d$ and odd $n$ (in particular, for the (4,3)-type of a spacetime) the VEVs of $\left\langle\phi^{2}\right\rangle_{\text {ren }}$ and $\left\langle T_{M N}\right\rangle_{\text {ren }}$ contain a logarithmic factor. In the pre-logarithmic coefficient we are in agreement with [5], and we have a discrepancy by a factor of 2 with [4]. Concerning the nonlogarithmic term in $\left\langle T_{M N}\right\rangle_{\text {ren }}$, we restrict its arbitrariness by a single arbitrary parameter, fixing the wider freedom in [4].

For the self-action, in addition to the four basic characteristic cases of parities $d$ and $n$, there is a significant exception of the monopole background ( $n=d-1)$. In most cases the self-action represents the Coulomb-like field with "charge" $\left(\xi-\xi_{d-1}\right)$ and vanishes for the particular value $\xi=\xi_{d-1}$ of the curvature coupling. Also it should be mentioned that (for $\xi=0$ ) the gravity-induced self-energy and the self-force of the point-like static electric charge $e$ can be obtained from our expressions by the formal identification $q^{2} \rightarrow e^{2}$, since the spacetime at hand is ultrastatic, and the defining expressions for the spatial scalar and the vectorial Green functions coincide.

We would like to emphasize that within our scheme, the appearance of the mass-dimension term inside the logarithm is related neither with the arbitrary scale factor $r_{0}$ coming from the Cartesian coordinates (2.5), nor with any length/mass of the problem at hand since the latter is absent. ${ }^{15}$ The logarithmic scale factor follows from the regularization (4.4) and its value, in principle, is arbitrary.

Making use of the same approach, but applying it to the delta-like interaction in the infinitely thin straight cosmic string, we have computed the effects under consideration. The results coincide with the ones in the literature [10-14,27] only for minimal and conformal coupling, while for other

\footnotetext{
15 For the real cosmic string one has its real width, but the results for the cosmic string within our model do not concern logarithms.
} 
values of $\xi$ they do not coincide already in the first order (in $\beta^{\prime}$ ). We refer this discrepancy to the missing of the $\xi$ correction inside the Green function. In the computation of $\left\langle T_{M N}\right\rangle_{\text {ren }}$ to the first order, this difference is reflected in terms $T_{M N}^{(0)}\left[\delta \mathcal{L}^{(\xi)}\right]$ and $T_{M N}^{(\xi)}\left[\delta \mathcal{L}^{(\xi)}\right]$. If to ignore these two in our scheme and retain the two remaining ones in (5.5), one would obtain the old answers.

We have shown that up to the first order in our Fourier transform language the results for the cosmic string spacetime can be obtained as a smooth limit of the corresponding results for a global monopole. From this framework, it represents a problem of independent interest whether this coincidence occurs only in the linear order in $\beta^{\prime}$, or if we here have the first non-vanishing part of the non-perturbative limit.

Finally, the usage of perturbation theory restricts the applicability by the requirement of the smallness of the angular deficit. However, this approach is relatively simple (to the order under consideration) and allows one to take advantage of the well-developed QFT methods. The Fourier transform allows one to consider all dimensional cases in the same language, as well as to include into consideration the delta-like potential for the cosmic string. This improves some drawback related with the coordinate-space Green functions, used in the cited literature, which on curved backgrounds differ drastically not only for the spacetimes of the opposite dimension parity, but it has no universal form even for the same-parity spacetimes. As a result, it allowed one to obtain and present in a closed, universal, and simple form the expressions valid for arbitrary $2 \leqslant n \leqslant(d-1)$ and $d \geqslant 3$, which, in its turn, verified the particular cases also, helping to justify (where we find confirmation) or correct/fix (where we do not) the corresponding well-known results.

Acknowledgements Yuri V. Grats thanks prof. A. V. Borisov for fruitful discussions. The work of Pavel Spirin is supported by the RFBR Grant 14-02-01092. Also PS acknowledges prof. T. N. Tomaras and grateful to the former non-profit "Dynasty" foundation (Russian Federation).

Open Access This article is distributed under the terms of the Creative Commons Attribution 4.0 International License (http://creativecomm ons.org/licenses/by/4.0/), which permits unrestricted use, distribution, and reproduction in any medium, provided you give appropriate credit to the original author(s) and the source, provide a link to the Creative Commons license, and indicate if changes were made.

Funded by SCOAP ${ }^{3}$.

\section{Appendix A: Basic integrals}

Here we give the derivation of basic integrals in the dimensional-regularization scheme we use. Such a scheme is rather common in QFT but unusual in the classical theory, so it may be instructive to briefly derive some useful integrals. The integrals are well defined for Euclidean prop- agators (with imaginary time), and analytically generalized for the case of Minkowski metric. Here we rewrite the Fourier transforms (3.13) in $d$ dimensions:

$\mathcal{F}\left[|\mathbf{r}|^{-\lambda}\right](k)=2^{d-\lambda} \pi^{d / 2} \frac{\Gamma\left(\frac{d-\lambda}{2}\right)}{\Gamma(\lambda / 2)} \frac{1}{|\mathbf{k}|^{d-\lambda}}$,

implying the Euclidean scalar products inside.

The scalar single-propagator integral is defined as

$J^{(1)}=\int \frac{d^{d} p}{(2 \pi)^{d}} \frac{1}{p^{2}-i \varepsilon}$.

Hereafter the right superscript labels the number of propagators. Passing to the spherical coordinates, one obtains the integral transformation with kernel $p^{n-3}$ acting on test function "1". Treating it as generalized function, in [42] it is shown that the latter equals zero in distributional sense, as well as

$J_{i_{1} \ldots i_{k}}^{(1)} \equiv \int \frac{d^{d} p}{(2 \pi)^{d}} \frac{p_{i_{1}} \ldots p_{i_{k}}}{p^{2}-i \varepsilon}=0$.

As is well known, this value is advocated as the absence of the parameter, upon which $J^{(1)}$ could depend explicitly, since the only variable $p$ in the integrand is integration one.

The scalar two-propagator integral is defined as

$J^{(2)}=\int \frac{d^{d} p}{(2 \pi)^{d}} \frac{1}{\left[p^{2}-i \varepsilon\right]\left[(p+q)^{2}-i \varepsilon\right]}$.

After the Wick rotation $p_{0}=i p_{E}, q_{0}=i q_{E}$ we have the analogous Euclidean integral. Thus consider the two following integrals with Euclidean scalar product:

$$
\begin{aligned}
J(q) & \equiv \int \frac{d^{d} p}{(2 \pi)^{d}} \frac{1}{p^{2}(p+q)^{2}}, \\
I & \equiv \int \frac{d^{d} p d^{d} q}{(2 \pi)^{2 d}} \frac{\mathrm{e}^{i x(p+q)}}{p^{2} q^{2}},
\end{aligned}
$$

with $J^{(2)}=i J(q)$, indeed. Being split on the product of identical integrals, $I$ equals

$I=\left(I_{0}\right)^{2}, \quad I_{0} \equiv \int \frac{d^{d} p}{(2 \pi)^{d}} \frac{\mathrm{e}^{i p x}}{p^{2}}$.

Making use of the Fourier transform (A.1), $I$ is given by

$I=\frac{1}{16 \pi^{d}} \frac{\Gamma^{2}\left(\frac{d-2}{2}\right)}{R^{2(d-2)}}, \quad R \equiv \sqrt{x^{2}}$.

Now we change variable $q \rightarrow p+q$ in $I$ (A.5):

$I=\int \frac{d^{d} p d^{d} q}{(2 \pi)^{2 d}} \frac{\mathrm{e}^{i x q}}{p^{2}(p+q)^{2}}=\mathcal{F}^{-1}[J(q)](x)$. 
Thus substituting (A.7) into (A.6) and taking the direct Fourier transform with the help of (A.1), $J^{(2)}(q)$ equals

$$
\begin{aligned}
J(q) & =\frac{\Gamma^{2}\left(\frac{d-2}{2}\right)}{16 \pi^{d}} \mathcal{F}\left[r^{-2(d-2)}\right](q) \\
& =\frac{\Gamma^{2}\left(\frac{d-2}{2}\right) \Gamma\left(-\frac{d-4}{2}\right)}{(4 \pi)^{d / 2} \Gamma(d-2)}|\mathbf{q}|^{d-4} .
\end{aligned}
$$

Restoring the Minkowskian $q^{0}$, after the $\Gamma$-function transformations, $J^{(2)}$ is given by

$$
J^{(2)}=-i \frac{2(d-1)}{(4 \pi)^{d / 2}} \frac{\Gamma^{2}(d / 2) \Gamma\left(-\frac{d-2}{2}\right)}{\Gamma(d)}\left(q^{2}\right)^{d / 2-2} .
$$

In the form (A.9) the arguments of all Gamma-functions do not intersect zero at $n \geqslant 3$. This result is identical to the ones given in a series of QFT textbooks and derived via Feynman parametrization, but remarkably, we have used just the single basic Fourier integral (A.1).

- The vectorial one reads

$$
J_{M}^{(2)}=\int \frac{d^{d} p}{(2 \pi)^{d}} \frac{p_{M}}{\left[p^{2}-i \varepsilon\right]\left[(p+q)^{2}-i \varepsilon\right]} .
$$

Obviously, the result has to be proportional to $q_{M}$ as to the only available input vector in the problem at hand: $J_{M}^{(2)}(q)=A_{1}(q) q_{M}$. Contracting this equality with $q^{M}$ and representing $p \cdot q=(1 / 2)\left[(p+q)^{2}-p^{2}-q^{2}\right]$, one uses (A.3) and (A.9) to determine the scalar $A_{1}$. Thus the result turns out to be

$$
\begin{aligned}
J_{M}^{(2)} & =-\frac{1}{2} J^{(2)} q_{M} \\
& =i \frac{d-1}{(4 \pi)^{d / 2}} \frac{\Gamma^{2}(d / 2) \Gamma\left(-\frac{d-2}{2}\right)}{\Gamma(d)}\left(q^{2}\right)^{d / 2-2} q_{M} .
\end{aligned}
$$

- The tensorial integral is defined as

$$
J_{M N}^{(2)}=\int \frac{d^{d} p}{(2 \pi)^{d}} \frac{p_{M} p_{N}}{\left[p^{2}-i \varepsilon\right]\left[(p+q)^{2}-i \varepsilon\right]} .
$$

Being a symmetric 2-rank tensor, the latter should be expressible via the flat metric $\eta_{M N}$ and $q_{M} q_{N}$ monomial: $J_{M N}^{(2)}=A_{2} q^{2} \eta_{M N}+A_{3} q_{M} q_{N}$. Taking the trace and substituting (A.3), one obtains the relation $n A_{2}+A_{3}=0$. Projecting (A.12) on $q^{N}$ and realizing the same strategy as before, one gets the second constraint:

$$
\left(A_{2}+A_{3}\right) q_{M}=\frac{1}{2} J_{M}^{(2)}=\frac{1}{4} q_{M} J^{(2)} .
$$

Resolving these two, the value of integral (A.12) is given by

$$
J_{M N}^{(2)}=-\frac{J^{(2)}}{4(d-1)}\left(q^{2} \eta_{M N}-d q_{M} q_{N}\right)
$$

with trace $\eta^{M N} J_{M N}^{(2)}=0$.

- Appealing to the same computational arguments, the three- and four-index integrals

$$
\begin{aligned}
J_{M N K}^{(2)} & \equiv \int \frac{d^{d} p}{(2 \pi)^{d}} \frac{p_{M} p_{N} p_{K}}{\left[p^{2}-i \varepsilon\right]\left[(p+q)^{2}-i \varepsilon\right]}, \\
J_{M N K L}^{(2)} & =\int \frac{d^{d} p}{(2 \pi)^{d}} \frac{p_{M} p_{N} p_{K} p_{L}}{\left[p^{2}-i \varepsilon\right]\left[(p+q)^{2}-i \varepsilon\right]},
\end{aligned}
$$

with the help of symmetry and some combinatorics, are given by

$$
\begin{aligned}
J_{M N K}^{(2)}= & -\frac{J^{(2)}}{8(d-1)}\left[(d+2) q_{M} q_{N} q_{K}\right. \\
& \left.-q^{2}\left(q_{M} \eta_{N K}+q_{N} \eta_{M K}+q_{K} \eta_{M N}\right)\right]
\end{aligned}
$$

and

$$
\begin{aligned}
J_{M N K L}^{(2)}= & \frac{J^{(2)}}{16\left(d^{2}-1\right)}\left[-(d+2) q^{2}\left(q_{M} q_{N} \eta_{K L}\right.\right. \\
& +q_{M} q_{K} \eta_{N L}+q_{M} q_{L} \eta_{N K} \\
& \left.+q_{N} q_{K} \eta_{M L}+q_{N} q_{L} \eta_{M K}+q_{K} q_{L} \eta_{M N}\right) \\
& +(d+4)(d+2) q_{M} q_{N} q_{K} q_{L}+\left(q^{2}\right)^{2} \\
& \left.\times\left(\eta_{M N} \eta_{K L}+\eta_{M K} \eta_{N L}+\eta_{M L} \eta_{N K}\right)\right],
\end{aligned}
$$

respectively.

\section{References}

1. P.C. Argyres, S. Dimopoulos, J. March-Russell, Phys. Lett. B 441, 96 (1998)

2. P. Meade, L. Randall, JHEP 0805, 003 (2008)

3. The CMS collaboration, JHEP 07, 178 (2013)

4. F.D. Mazzitelli, C.O. Lousto, Phys. Rev. D 43, 468 (1991)

5. E.R. Bezerra de Mello, Phys. Rev. D 73, 105015 (2006)

6. E.R. Bezerra de Mello, J. Math. Phys. 43, 1018 (2002)

7. E.R. Bezerra de Mello, Phys. Rev. D 76, 125021 (2007)

8. D. Barbosa, U. de Freitas, E.R. Bezerra de Mello, Class. Quantum Gravity 28, 065009 (2011)

9. E.R. Bezerra de Mello, A.A. Saharian, Class. Quantum Gravity 29, 135007 (2012)

10. J.S. Dowker, Phys. Rev. D 36, 3742 (1987)

11. T.M. Helliwell, D.A. Konkowski, Phys. Rev. D 34, 1918 (1986) 
12. V.P. Frolov, E.M. Serebriany, Phys. Rev. D 35, 3779 (1987)

13. T. Souradeep, V. Sahni, Phys. Rev. D 46, 1616 (1992)

14. B. Linet, Phys. Rev. D 33, 1833 (1986)

15. G.W. Gibbons, S.W. Hawking, T. Vachaspati (eds.), The Formation and Evolution of Cosmic Strings (Cambridge University Press, Cambridge, 1990)

16. A. Vilenkin, E.P.S. Shellard, Cosmic Strings and Other Topological Defects (Cambridge University Press, Cambridge, 1994)

17. D.D. Sokolov, A.A. Starobinsky, Sov. Phys. Dokl. 22, 312 (1977)

18. S. Deser, R. Jackiw, G. 't Hooft, Ann. Phys. NY 152, 220 (1984)

19. J.R. Gott, M. Alpert, Gen. Relativ. Gravit. 16, 243 (1984)

20. S. Giddings, J. Abbott, K. Kuchnar, Gen. Relativ. Gravit. 16, 751 (1984)

21. R. Jackiw, Nucl. Phys. B 252, 343 (1985)

22. P.C. Letelier, Class. Quantum Gravity 4, 75 (1987)

23. Yu.V. Grats, A. Garcia, Class. Quantum Gravity 13, 189 (1996)

24. E.R. Bezerra de Mello, V.B. Bezerra, YuV Grats, Mod. Phys. Lett. A. 13(18), 1427 (1998)

25. E.R. Bezerra de Mello, V.B. Bezerra, YuV Grats, Class. Quantum Gravity 15, 1915 (1998)

26. Yu.V. Grats, A.A. Rossikhin, Theor. Math. Phys. 123, 539 (2000)

27. D.V. Gal'tsov, Yu.V. Grats, A.B. Lavrent'ev, Phys. Atom. Nucl. 58, $516(1995)$

28. M. Bariola, A. Vilenkin, Phys. Rev. Lett. 63, 341 (1989)

29. D. Harari, C. Lousto, Phys. Rev. D 42, 2626 (1990)

30. A. Vilenkin, Phys. Rev. D 23, 852 (1981)

31. J.R. Gott, Astrophys. J. 288, 422 (1985)

32. J.S. Dowker, Phys. Rev. D 36, 3095 (1987)

33. I. Olasagasti, A. Vilenkin, Phys. Rev. D 62, 044014 (2000)

34. J. Spinelly, E.R. Bezerra de Mello, JHEP 09, 005 (2008)
35. J. Spinelly, E.R. Bezerra de Mello, Int. J. Mod. Phys. A. 24, 1481 (2009)

36. Yu.V. Grats, Theor. Math. Phys. 186, 205 (2016)

37. E.R. Bezerra de Mello, Class. Quantum Gravity 27, 095017 (2010)

38. E.A.F. Bragança, H.F.S. Mota, E.R. Bezerra de Mello, Int. J. Mod. Phys. D 24, 1550055 (2015)

39. E.R. Bezerra de Mello et al., Phys. Rev. D 91, 064034 (2015)

40. A.G. Smith, C.M. Will, Phys. Rev. D 22, 1276 (1980)

41. J. Schwinger, Phys. Rev. 82, 664 (1951)

42. I.M. Gel'fand, G.E. Shilov, Generalized Functions: Properties and Operations (Academic Press, Waltham, 1964)

43. J.C. Collins, Renormalization (Cambridge University Press, Cambridge, 1984)

44. S.W. Hawking, Commun. Math. Phys. 55, 133 (1977)

45. V.A. Smirnov, Analytic Tools for Feynman Integrals, Springer Tracts in Modern Physics, vol. 250 (Springer, Berlin, 2012)

46. N.D. Birrell, P.C.W. Davies, Quantum Fields in Curved Space (Cambridge University Press, Cambridge, 1982)

47. M.A. Castagnino, D.D. Harari, C.A. Nuñez, J. Math. Phys. 28, 184 (1987)

48. S.M. Christensen, Phys. Rev. D 14, 2490 (1976)

49. S.M. Christensen, Phys. Rev. D 17, 946 (1978)

50. E.R. Bezerra de Mello, C. Furtado, Phys. Rev. D 56, 1345 (1997)

51. S. Albeverio et al., Solvable Models in Quantum Mechanics, 2nd edn. (AMS Chelsea Publishing, Providence, 2004)

52. R.W. Jackiw, Divers Topics in Theoretical and Mathematical Physics, Advanced Series in Mathematical Physics (World Scientific, Singapore, 1995)

53. I. Jack, L. Parker, Phys. Rev. D 31, 2439 (1985) 